\title{
ON A DIPHASIC LOW MACH NUMBER SYSTEM
}

\author{
StéPhane Dellacherie ${ }^{1,2}$
}

\begin{abstract}
We propose a Diphasic Low Mach Number (DLMN) system for the modelling of diphasic flows without phase change at low Mach number, system which is an extension of the system proposed by Majda in [Center of Pure and Applied Mathematics, Berkeley, report No. 112] and [Combust. Sci. Tech. 42 (1985) 185-205] for low Mach number combustion problems. This system is written for $a$ priori any equations of state. Under minimal thermodynamic hypothesis which are satisfied by a large class of generalized van der Waals equations of state, we recover some natural properties related to the dilation and to the compression of bubbles. We also propose an entropic numerical scheme in Lagrangian coordinates when the geometry is monodimensional and when the two fluids are perfect gases. At last, we numerically show that the DLMN system may become ill-posed when the entropy of one of the two fluids is not a convex function.
\end{abstract}

Mathematics Subject Classification. 35Q30, 65M12, 76T10, 80A10.

Contributed talk, Low Mach Number Flows Conference, June 21-25, 2004, Porquerolles, France.

\section{INTRODUCTION}

In the field of the numerical simulation of nuclear reactors, the Direct Numerical Simulation (DNS) of diphasic flows is an important research topic. Indeed, in a core of a PWR reactor (Pressurized Water Reactor) in France or of a CANDU reactor (CANadian Deuterium Uranium) in Canada, the water exists under the liquid phase and under the vapor phase, and it is a difficult task to propose macroscopic modellings - i.e. at the scale of a reactor - because of the complex structure of such diphasic flows at the mesoscopic scale - i.e. at the scale of vapor bubbles -. Coupled to experimental studies [20], the DNS of such diphasic flows at the mesoscopic scale may be a way to better understand these kind of complex flows at the macroscopic scale and, thus, to propose or to justify in the future closure laws for diphasic modellings at the scale of a reactor. Of course, it already exists DNS of diphasic flows. For example, in $[10,15,17,19,24,28,30]$, incompressible diphasic flows are studied; in $[1-3,16,18,23]$, simulations of compressible diphasic flows are proposed. In our case, despite the Mach number is very low, we cannot use a priori any incompressible model because:

- there is high heat transfers between each phase;

- one of the phase may be more compressible than the other (gas versus liquid for example);

- it may exist phase change phenomena.

\footnotetext{
Keywords and phrases. Diphasic flow, low mach number system, thermodynamic equilibrium, entropy, van der Waals equations of state.

1 Commissariat à l'Énergie Atomique, 91191 Gif sur Yvette, France.

2 Centre de Recherches Mathématiques, Université de Montréal, C.P. 6128-succursale Centre-ville, Montréal, H3C 3J7, Canada. stephane.dellacherie@cea.fr; dellache@crm.umontreal.ca 
Indeed, it would be impossible to take into account such phenomena without any information coming from the equations of state of each phase. Thus, it seems that without any additional modelling hypothesis, the compressible diphasic Navier-Stokes system should be as in [14] the right model for our DNS. Nevertheless, the numerical discretisation of the compressible Navier-Stokes system is a very difficult task when the Mach number goes to zero because of the existence of two different time scales (a first one linked to the material waves and a second one linked to the acoustic waves whose celerity is very high when the Mach number is low: see for example $[11,12])$. Since we suppose that the Mach number is always close to zero - i.e. we are not interested in transonic flows -, we propose to bypass this difficulty by filtering out the acoustic waves directly in the compressible diphasic Navier-Stokes system through a formal asymptotic expansion. This means that we do not modify any compressible diphasic numerical scheme by applying an ad hoc preconditioning technic as in $[11,12]$ where transonic flows are considered (see also $[4,29]$ ). As a consequence, the thermodynamic pressure deduced from this Diphasic Low Mach Number (DLMN) system is homogeneous in space which means that this pressure is an average thermodynamic pressure. Let us note that this filter out technic was previously proposed by Majda in [21,22] for low Mach number combustion problems (see also [25] for a monophasic perfect gas).

In this paper, we will see that the DLMN system has good properties. For example, it predicts the dilation or the compression of a bubble under minimal thermodynamic hypothesis (which are satisfied by a large class of generalized van der Waals equations of state) and we verify that the entropy is a central quantity to obtain the existence of an equilibrium when the time goes to infinity. Moreover, we formally prove that the DLMN system degenerates toward the incompressible Navier-Stokes system for one of the fluids under modelling hypothesis (which can be satisfied by ad hoc generalized van der Waals equations of state). Let us note that we do not take into account in that paper any phase change phenomena - i.e we focus on the non-miscible charactere of the diphasic flow - and we neglect any influence of the surface tension despite they are central phenomena in the field of diphasic flows. At last, we do not study any well-posedeness properties of the DLMN system in appropriate Sobolev spaces: this important question will be studied in a future work by applying the technic of Embid [9].

The plan of this paper is the following: In the second section, we propose a formal derivation of the DLMN system inspired of [22]. In the third section, we describe basic properties of the DLMN system. In the fourth section, we describe the Lagrangian formulation of the DLMN system when each fluid is a perfect gas and when the geometry is monodimensional. In the fifth section, we propose an entropic scheme in monodimensional geometry. In the sixth section, we present numerical results (see also [8]).

\section{The Diphasic Low Mach Number system}

In this section, we propose a formal derivation of the Diphasic Low Mach Number (DLMN) system using an asymptotic expansion applied to the compressible diphasic Navier-Stokes system written in the non-conservative form

$$
\left\{\begin{array}{l}
D_{t} Y_{1}=0 \\
\partial_{t} \rho+\nabla \cdot(\rho u)=0 \\
\rho D_{t} u=-\nabla P+\nabla \cdot \sigma-\rho g \\
\rho C_{p} D_{t} T=\alpha T D_{t} P+\nabla \cdot q+\sigma: \nabla u
\end{array}\right.
$$

This formal derivation is inspired from the paper [22] of Majda and Sethian where it is proposed a low Mach number system for the modelling of combustion of perfect gases (see also [25] in the case of a monophasic flow for perfect gases).

In the system (1), $t \geq 0$ is the time variable, $x \in \Omega \subset \mathbb{R}^{d}(d=1,2$ or 3$)$ is the space variable and $D_{t}=\partial_{t}+u \cdot \nabla$ is the Lagrangian derivative operator. The function $Y_{1}(t, x)$ takes its values in $\{0,1\}$ knowing that the initial 
condition for $Y_{1}(t, x)$ is given by

$$
Y_{1}(t=0, x)=\left\{\begin{array}{lll}
1 & \text { if } \quad x \in \Omega_{1}(t=0) & \text { (i.e. fluid 1) }, \\
0 & \text { if } \quad x \in \Omega_{2}(t=0) & \text { (i.e. fluid 2), }
\end{array}\right.
$$

$\Omega_{k}(t=0) \neq \emptyset$ defining the initial topology of the flow $(k \in\{1,2\})$. Let us remark that (1)(a) and (2) impose that for any $(t, x), Y_{1}(t, x) \in\{0,1\}$. Thus, we can define the two domains $\Omega_{1}(t)$ and $\Omega_{2}(t)$ at any time $t \geq 0$ with $\Omega_{1}(t)=\left\{x \in \Omega\right.$ such that $\left.Y_{1}(t, x)=1\right\}$ and $\Omega_{2}(t)=\left\{x \in \Omega\right.$ such that $\left.Y_{1}(t, x)=0\right\}$. The function $Y_{1}(t, x)$ can be seen as the color function or as the volumic fraction of the fluid 1 whose discontinuity surface $\Sigma(t)=\partial \Omega_{1}(t) \cap \partial \Omega_{2}(t)$ localizes at any time $t \geq 0$ the interface between the fluid 1 and the fluid 2 (thus, we have $\left.\Omega=\Omega_{1}(t) \cup \Omega_{2}(t) \cup \Sigma(t)\right)$. The vector $g=9,81 \hat{z} \mathrm{~m} \cdot \mathrm{s}^{-2}$ is the gravity $(\hat{z}$ is the unitary vector in the vertical direction). The quantities $\rho\left(Y_{1}, T, P\right), T, P$ and $u$ are respectively the density, the temperature, the thermodynamic pressure and the fluid velocity at the time-space point $(t, x)$. The viscosity tensor $\sigma$ and the Fourier heat flux $q$ are defined with

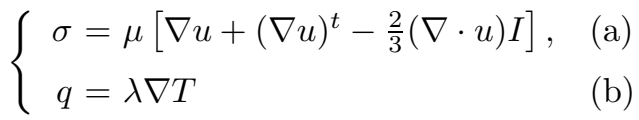

( $I$ is the $d \times d$ unitary matrix) where $\mu\left(Y_{1}, T, P\right)$ and $\lambda\left(Y_{1}, T, P\right)$ are respectively the fluid viscosity and the thermal conductivity. The quantities $\alpha\left(Y_{1}, T, P\right)$ and $C_{p}\left(Y_{1}, T, P\right)$ are respectively the compressibility coefficient at constant pressure (also called thermal or volumic expansion coefficient) and the calorific capacity at constant pressure. They are given by

and by

$$
\alpha\left(Y_{1}, T, P\right)=-\frac{1}{\rho} \cdot \frac{\partial \rho}{\partial T}\left(Y_{1}, T, P\right)
$$

$$
C_{p}\left(Y_{1}, T, P\right)=\frac{\partial h}{\partial T}\left(Y_{1}, T, P\right)
$$

where $h \equiv \varepsilon+P / \rho$ is the enthalpy, $\varepsilon\left(Y_{1}, T, P\right)$ being the internal energy of the fluid. Because of the non-miscible charactere of the diphasic flow, all the thermodynamic quantities $\xi \in\left\{\mu, \lambda, \alpha, C_{p}, \rho, \varepsilon\right\}$ are given by

$$
\xi\left(Y_{1}, T, P\right)=Y_{1} \xi_{1}(T, P)+\left(1-Y_{1}\right) \xi_{2}(T, P)
$$

knowing that $Y_{1}(t, x) \in\{0,1\}$. The functions $\rho_{k}(T, P)$ and $\varepsilon_{k}(T, P)$ define the equations of state of the fluid $k$. Let us note that we suppose that each fluid verifies the thermodynamic hypothesis:

Hypothesis 2.1. The equations of state $\rho_{k}(T, P) \equiv 1 / \tau_{k}(T, P)$ and $\varepsilon_{k}(T, P)$ of each fluid $k \in\{1,2\}$ are such that it exists a function $s_{k}\left(\tau_{k}, \varepsilon_{k}\right)$ verifying

$$
\begin{cases}s_{k}\left(\tau_{k}, \varepsilon_{k}\right) \text { is a strictly convex function, } & (\text { a }) \\ -T d s_{k}=d \varepsilon_{k}+P d \tau_{k} . & (b)\end{cases}
$$

Of course, the function $s_{k}\left(\tau_{k}, \varepsilon_{k}\right)$ is the classical thermodynamic entropy of the fluid $k$. This hypothesis is sufficient to write that all the thermodynamic quantities can be written in function of $\left(Y_{1}, T, P\right)$ (for example, we deduce the expression (4) for $\alpha$ when this hypothesis is valid).

At last, the boundary conditions for the system (1) are defined with

$$
\left\{\begin{aligned}
\forall x \in \Sigma(t): \quad & \left.u\right|_{\Sigma_{1}(t)}=\left.u\right|_{\Sigma_{2}(t)} \\
& \left.\sigma\right|_{\Sigma_{1}(t)} \cdot n_{1 \rightarrow 2}=\left.\sigma\right|_{\Sigma_{2}(t)} \cdot n_{1 \rightarrow 2} \\
& \left.T\right|_{\Sigma_{1}(t)}=\left.T\right|_{\Sigma_{2}(t)} \\
& \left.\lambda_{1} \nabla T\right|_{\Sigma_{1}(t)} \cdot n_{1 \rightarrow 2}=\left.\lambda_{2} \nabla T\right|_{\Sigma_{2}(t)} \cdot n_{1 \rightarrow 2}
\end{aligned}\right.
$$


and with

$$
\left\{\begin{array}{lll}
\forall x \in \partial \Omega: & u(t, x)=0, & \text { (a) } \\
& \nabla T(t, x) \cdot n(x)=0 . & \text { (b) }
\end{array}\right.
$$

The notation $\left.\phi\right|_{\Sigma_{k}(t)}$ corresponds to the extension on the surface $\Sigma(t)$ of the restriction of the function $\phi(t, x)$ to the open domain $\Omega_{k}(t)$. The vector $n_{1 \rightarrow 2}$ is the unitary normal vector to the surface $\Sigma(t)$ oriented to the fluid 2. The vector $n$ is the unitary vector to the surface $\partial \Omega$ oriented to the exterior of $\Omega$.

\subsection{Non-dimensioned formulation}

To obtain the non-dimensioned formulation of (1), we suppose that the five following hypothesis are verified:

Hypothesis 2.2. The diphasic flow is such that it is possible to define a characteristic length $L_{*}$ and a characteristic velocity $u_{*}$ common to the domains $\Omega_{1}(t)$ and $\Omega_{2}(t)$. Thus, we define the characteristic time $t_{*}$ with $L_{*}=u_{*} \cdot t_{*}$.

Hypothesis 2.3. It is possible to define a characteristic temperature $T_{*}$ and a characteristic pressure $P_{*}$ common to the fluids 1 and 2 .

Hypothesis 2.4. The fluids 1 and 2 have similar equations of state $\rho_{k}(T, P)$ and $\varepsilon_{k}(T, P)$ and similar transport coefficients $\mu_{k}$ and $\lambda_{k}$.

Hypothesis 2.5. The equations of state of the fluid 1 are such that we can define a characteristic value $C_{p *, 1}$ for $C_{p, 1}$ such that $\frac{u_{*}^{2}}{T_{*} C_{p *, 1}}=M_{*}^{2}$ where $M_{*}=\frac{\left|u_{*}\right|}{\sqrt{P_{*} / \rho_{*, 1}}}$ is the Mach number in the fluid $1\left(\rho_{*, 1}=\rho_{1}\left(T_{*}, P_{*}\right)\right)$. Let us remark that this relation is equivalent to $T_{*} C_{p *, 1}=P_{*} / \rho_{*, 1}$.

Hypothesis 2.6. The flow verifies $M_{*} \ll 1$.

The Hypothesis 2.2 means that, through the asymptotic expansion that we will expose below, we will filter out any phenomena whose characteristic time is very small compared to the characteristic time $L_{*} / u_{*}$. The Hypothesis 2.3 is necessary: it means that the thermodynamic state in $\Omega(t)$ characterized by $(T, P)$ will vary in a "reasonable" bounded domain. The Hypothesis 2.4 allows to define characteristic values $\rho_{*}=\rho_{*, 1}, C_{p *}=C_{p *, 1}$, $\mu_{*}=\mu_{*, 1}$ and $\lambda_{*}=\lambda_{*, 1}$ for $\rho, C_{p}, \mu$ and $\lambda$ which are representative for the fluids 1 and 2 . Let us note that it is possible to derive the DLMN system without the Hypothesis 2.4 (see Chap. 1 of [7]): we take into account this hypothesis in this section to simplify the formal derivation. The Hypothesis 2.5 restricts the kind of equations of state for the fluid 1: we can say that when the fluid 1 is close to a perfect gas, the Hypothesis 2.5 is verified. At last, the Hypothesis 2.6 means that we study diphasic flows which are highly subsonic in the fluid 1 and in the fluid 2 .

Under the Hypothesis 2.2 to 2.5, we obtain the classical non-dimensioned system

$$
\left\{\begin{array}{l}
D_{t} Y_{1}=0 \\
\partial_{t} \rho+\nabla \cdot(\rho u)=0 \\
\rho D_{t} u=-\frac{\nabla P}{M_{*}^{2}}+\frac{\nabla \cdot \sigma}{R e_{*}}-\frac{\rho}{F r_{*}} \hat{z}, \\
\rho C_{p} D_{t} T=\beta_{*} \cdot \alpha T D_{t} P+\frac{\nabla \cdot(\lambda \nabla T)}{R e_{*} P r_{*}}+\frac{M_{*}^{2}}{R e_{*}} \sigma: \nabla u,
\end{array}\right.
$$


where the non-dimensioned numbers are given by

$$
\left\{\begin{array}{l}
R e_{*}=\frac{u_{*} L_{*} \rho_{*}}{\mu_{*}} \quad \text { (Reynolds number) } \\
P r_{*}=\frac{\mu_{*} C_{p *}}{\lambda_{*}} \quad \text { (Prandtl number) } \\
\left.F r_{*}=\frac{u_{*}^{2}}{L_{*} g_{*}} \quad \text { (Froude number }\right), \\
\beta_{*}=\frac{\alpha_{*} P_{*}}{\rho_{*} C_{p *}}
\end{array}\right.
$$

Let us note that under the Hypothesis 2.5, we find that $\beta_{*}=\alpha_{*} T_{*}$. And, since it is easy to verify that $\alpha=1 / T$ when the fluids are perfect gases, we find that $\beta_{*}=1$ is the good choice in the case of perfect gases.

\subsection{Formal asymptotic expansion}

Under the Hypothesis 2.6, we suppose that the solution of (10) can be expanded with

$$
\left\{\begin{array}{l}
Y_{1}(t, x)=Y_{1}^{0}(t, x)+Y_{1}^{1}\left(t, x, M_{*}\right), \\
\Phi(t, x)=\Phi^{0}(t, x)+M_{*} \Phi^{1}\left(t, x, M_{*}\right)+M_{*}^{2} \Phi^{2}(t, x)+\mathcal{O}\left(M_{*}^{3}\right),
\end{array}\right.
$$

where $\Phi \equiv(u, \rho, T, P)$. The function $Y_{1}^{1}\left(t, x, M_{*}\right)$ is a function which takes its values in $\{0,1\}$ whose support measure is supposed to converge to zero when $M_{*}$ goes to zero. Let us note that by supposing that the equations of state are regular functions of $(T, P)$, we obtain that $\rho^{0}(t, x)=\rho\left[Y_{1}^{0}(t, x), T^{0}(t, x), P^{0}(t, x)\right]$ where $\rho$ is given by $(6)$. By injecting the expansion (12) in (10), we formally find that

$$
\nabla P^{0}(t, x)=0 \quad \text { and } \quad \nabla P^{1}\left(t, x, M_{*}\right)=0
$$

when $M_{*}$ goes to zero. This allows to write that $D_{t} P^{0}(t, x)=P^{0^{\prime}}(t)$. We also find that

$$
\left\{\begin{array}{l}
D_{t} Y_{1}^{0}=0 \\
\partial_{t} \rho^{0}+\nabla \cdot\left(\rho^{0} u^{0}\right)=0 \\
\rho^{0} C_{p}^{0} D_{t} T^{0}=\beta_{*} \alpha^{0} T^{0} D_{t} P^{0}+\frac{\nabla \cdot\left(\lambda^{0} \nabla T^{0}\right)}{R e_{*} P r_{*}}
\end{array}\right.
$$

with $D_{t}=\partial_{t}+u^{0} \cdot \nabla$ and with $\left(C_{p}^{0}, \alpha^{0}\right)(t, x)=\left(C_{p}, \alpha\right)\left[Y_{1}^{0}(t, x), T^{0}(t, x), P^{0}(t, x)\right]$. Moreover, the equation $(10)(\mathrm{c})$ shows that

$$
\mathcal{P}\left(\rho D_{t} u-\frac{\nabla \cdot \sigma}{R e_{*}}+\frac{\rho}{F r_{*}} \hat{z}\right)=0
$$

where $\mathcal{P}$ is the Hodge projection on the free divergence vector space. Thus, by injecting the asymptotic expansion (12) in (15), we also find that

$$
\mathcal{P}\left(\rho^{0} D_{t} u^{0}-\frac{\nabla \cdot \sigma^{0}}{R e_{*}}+\frac{\rho^{0}}{F r_{*}} \hat{z}\right)=0
$$

which implies that it exists a function $\Pi(t, x)$ independent of $M_{*}$ and homogeneous to a pressure such that

$$
\rho^{0} D_{t} u^{0}=-\nabla \Pi+\frac{\nabla \cdot \sigma^{0}}{R e_{*}}-\frac{\rho^{0}}{F r_{*}} \hat{z} .
$$


Finally, the vector $\left(Y_{1}^{0}, u^{0}, \rho^{0}, T^{0}, P^{0}\right)$ - that we note $\left(Y_{1}, u, \rho, T, P\right)$ to simplify the notations - is solution of the system

$$
\begin{cases}D_{t} Y_{1}=0, & \text { (a) } \\ \rho C_{p} D_{t} T=\beta_{*} \cdot \alpha T P^{\prime}(t)+\frac{\nabla \cdot(\lambda \nabla T)}{R e_{*} P r_{*}}, & \text { (b) } \\ \partial_{t} \rho+\nabla \cdot(\rho u)=0, & \text { (c) } \\ \rho D_{t} u=-\nabla \Pi(t, x)+\frac{\nabla \cdot \sigma}{R e_{*}}-\frac{\rho}{F r_{*}} \hat{z}, & \text { (d) }\end{cases}
$$

where $\rho\left(Y_{1}, T, P\right)$ is defined with (6). Thus, we have 4 equations for the 5 independent unknowns $Y_{1}, u, T, P$ and $\Pi$.

The last equation is obtained by finding an equation whose the solution is $P(t)$, equation which is directly related to the boundary conditions (8)-(9) and, thus, to the shape of the interface $\Sigma(t)$. First of all, let us come back to the dimensioned system (1) and let us remark that the continuity equation (1)(b) is equivalent to $\nabla \cdot u=G$ with

$$
G(t, x) \equiv-\frac{D_{t} \rho}{\rho}\left(Y_{1}, T, P\right)=-\frac{1}{\rho}\left(\frac{\partial \rho}{\partial Y_{1}} D_{t} Y_{1}+\frac{\partial \rho}{\partial T} D_{t} T+\frac{\partial \rho}{\partial P} D_{t} P\right)
$$

where the various variables are given their initial dimensioned meaning. By using the equations (1)(a)(d), we obtain that

$$
G(t, x)=\frac{D_{t} P}{\rho} \cdot\left(\frac{\alpha^{2} T}{C_{p}}-\frac{\partial \rho}{\partial P}\right)+\frac{\beta}{P} \nabla \cdot(\lambda \nabla T)+\frac{\beta}{P} \sigma: \nabla u,
$$

where the non-dimensioned thermodynamic function $\beta\left(Y_{1}, T, P\right)$ is defined with

$$
\beta\left(Y_{1}, T, P\right)=\frac{\alpha P}{\rho C_{p}}\left(Y_{1}, T, P\right)
$$

(let us remark that the function $\beta\left(Y_{1}, T, P\right)$ is linked to the Grüneisen function: see Sect. 3.2). Let us recall that, under the thermodynamic hypothesis 2.1 , we have the strict positivity of the quantity $\frac{\partial \rho}{\partial P}-\frac{\alpha^{2} T}{C_{p}}$ and that the sound velocity $c\left(Y_{1}, T, P\right)$ can be defined in function of $\alpha$ and $C_{p}$ with

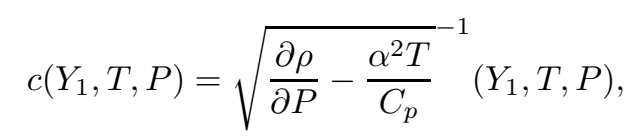

which implies that

$$
G(t, x)=-\frac{D_{t} P}{\rho c^{2}}+\frac{\beta}{P} \nabla \cdot(\lambda \nabla T)+\frac{\beta}{P} \sigma: \nabla u
$$

By coming back to the non-dimensioned variables, we find that

$$
\nabla \cdot u=-\frac{1}{\Gamma_{*}} \cdot \frac{D_{t} P}{\Gamma P}+\frac{\beta_{*}}{R e_{*} P r_{*}} \cdot \frac{\beta}{P} \nabla \cdot(\lambda \nabla T)+\beta_{*} \frac{M_{*}^{2}}{R e_{*}} \cdot \frac{\beta}{P} \sigma: \nabla u,
$$

where $\Gamma\left(Y_{1}, T, P\right)$ is defined with

$$
\Gamma\left(Y_{1}, T, P\right)=\frac{\rho c^{2}}{P}\left(Y_{1}, T, P\right),
$$

$\Gamma_{*}$ being a characteristic value of $\Gamma$ (let us remark that for a perfect gas, $\Gamma$ is equal to the classical constant $\gamma=\mathcal{O}(1)$ which implies that we can take $\Gamma_{*}=1$ in that case). By injecting the asymptotic expansion (12) in (20) and by only keeping the terms of order zero, we find that

$$
\nabla \cdot u=G(t, x)
$$


with now

$$
G(t, x)=-\frac{1}{\Gamma_{*}} \cdot \frac{P^{\prime}(t)}{\Gamma P(t)}+\frac{\beta_{*}}{R e_{*} P r_{*}} \cdot \frac{\beta}{P(t)} \nabla \cdot(\lambda \nabla T)
$$

(knowing that $P=P(t))$ where the vector $(u, T, \ldots)$ relates to $\left(u^{0}, T^{0}, \ldots\right)$ for sake of simplicity. The formulae (22)-(23) allow us to obtain the lemma:

Lemma 2.1. Under the boundary conditions (8)-(9), $P(t)$ is solution of the (non-dimensioned) non-linear integro-differential equation

$$
\begin{aligned}
P(t)^{\prime} & =\frac{\beta_{*} \Gamma_{*}}{R e_{*} P r_{*}} \cdot \frac{\int_{\Omega(t)} \beta\left(Y_{1}, T, P\right) \nabla \cdot \lambda \nabla T \mathrm{~d} x}{\int_{\Omega} \frac{\mathrm{d} x}{\Gamma\left(Y_{1}, T, P\right)}} \\
& =\frac{\beta_{*} \Gamma_{*}}{R e_{*} P r_{*}} \cdot\left\{\frac{\int_{\Sigma(t)}[\beta]_{\Sigma(t)}(T, P) \cdot \lambda \nabla T \cdot n_{1 \rightarrow 2} \mathrm{~d} \Sigma}{\int_{\Omega} \frac{\mathrm{d} x}{\Gamma\left(Y_{1}, T, P\right)}}-\frac{\int_{\Omega} \frac{\partial \beta}{\partial T}\left(Y_{1}, T, P\right) \lambda\left(Y_{1}, T, P\right)(\nabla T)^{2} \mathrm{~d} x}{\int_{\Omega} \frac{\mathrm{d} x}{\Gamma\left(Y_{1}, T, P\right)}}\right\}
\end{aligned}
$$

with $\left.[\beta]_{\Sigma}(T, P) \equiv \beta_{1}(T, P)\right|_{\Sigma}-\left.\beta_{2}(T, P)\right|_{\Sigma}$.

Let us note that the first term in the right hand side of $(24)(\mathrm{b})$ is directly related to the diphasic and non-miscible charactere of the flow and that the second term takes into account the fact that one of the two fluids may not be a perfect gas. Indeed, when the two fluids are perfect gases, we have $\partial_{T} \beta\left(Y_{1}, T, P\right)=0$ : see Section 4. This explains why this term does not exist in the monophasic low Mach number model for perfect gases presented in $[21,22,25]$.

Proof of lemma 2.1. By applying the Hodge decomposition to the velocity field $u(t, x)$, we find that the potential part $\nabla \phi(t, x)$ of $u(t, x)$ verifies the Neumann elliptic problem

$$
\left\{\begin{array}{l}
\Delta \phi(t, x)=G(t, x), \\
\left.\nabla \phi(t, x)\right|_{\partial \Omega} \cdot n(x)=0
\end{array}\right.
$$

$(n(x)$ is the normal vector to $\partial \Omega)$. And, by using the Fredholm alternative, we obtain that the elliptic equation (25) admits an unique solution (up to a constant) if and only if

$$
\int_{\Omega} G(t, x) \mathrm{d} x=0
$$

which corresponds to the Neumann compatibility condition (cf. [5] p. 51-52). This last relation coupled to (23) induces that

$$
\begin{aligned}
P^{\prime}(t) & =\frac{\beta_{*} \Gamma_{*}}{R e_{*} P r_{*}} \cdot \sum_{k=1,2} \frac{\int_{\Omega_{k}(t)} \beta_{k}(T, P) \nabla \cdot(\lambda \nabla T) \mathrm{d} x}{\int_{\Omega} \frac{\mathrm{d} x}{\Gamma\left(Y_{1}, T, P\right)}} \\
& =\frac{\beta_{*} \Gamma_{*}}{R e_{*} P r_{*}} \cdot\left\{\frac{\int_{\Sigma(t)}[\beta]_{\Sigma(t)}(T, P) \lambda \nabla T \cdot n_{1 \rightarrow 2} \mathrm{~d} \Sigma}{\int_{\Omega} \frac{\mathrm{d} x}{\Gamma\left(Y_{1}, T, P\right)}}-\frac{\int_{\Omega} \nabla \beta\left(Y_{1}, T, P\right) \cdot \lambda \nabla T \mathrm{~d} x}{\int_{\Omega} \frac{\mathrm{d} x}{\Gamma\left(Y_{1}, T, P\right)}}\right\} .
\end{aligned}
$$


We conclude by noting that $\nabla \beta\left(Y_{1}, T, P\right)=\frac{\partial \beta}{\partial Y_{1}} \nabla Y_{1}+\frac{\partial \beta}{\partial T} \nabla T+\frac{\partial \beta}{\partial P} \nabla P$ and that

$$
\left\{\begin{array}{l}
\nabla Y_{1}=0 \quad \text { in } \quad \Omega_{k}(t) \\
\nabla P(t)=0
\end{array}\right.
$$

\subsection{Final formulation of the DLMN system}

By summarizing the previous results, we obtain that the dimensioned DLMN system is constituted with the two coupled systems

$$
\left\{\begin{array}{l}
D_{t} Y_{1}=0 \\
\rho C_{p} D_{t} T=\alpha T P^{\prime}(t)+\nabla \cdot q
\end{array}\right.
$$

and

$$
\left\{\begin{array}{l}
\nabla \cdot u=G \\
\rho D_{t} u=-\nabla \Pi+\nabla \cdot \sigma-\rho g \hat{z},
\end{array}\right.
$$

knowing that the function $G(t, x)$ is given by

$$
G(t, x)=-\frac{1}{\Gamma} \cdot \frac{P^{\prime}(t)}{P(t)}+\frac{\beta}{P(t)} \nabla \cdot q
$$

and that the pressure $P(t)$ is solution of the integro-differential equation

$$
\begin{aligned}
P^{\prime}(t) & =\frac{\int_{\Omega(t)} \beta\left(Y_{1}, T, P\right) \nabla \cdot \lambda \nabla T \mathrm{~d} x}{\int_{\Omega} \frac{\mathrm{d} x}{\Gamma\left(Y_{1}, T, P\right)}} \\
& =\frac{\int_{\Sigma(t)}[\beta]_{\Sigma(t)}(T, P) \lambda \nabla T \cdot n_{1 \rightarrow 2} \mathrm{~d} \Sigma}{\int_{\Omega} \frac{\mathrm{d} x}{\Gamma\left(Y_{1}, T, P\right)}}-\frac{\int_{\Omega} \frac{\partial \beta}{\partial T}\left(Y_{1}, T, P\right) \lambda\left(Y_{1}, T, P\right)(\nabla T)^{2} \mathrm{~d} x}{\int_{\Omega} \frac{\mathrm{d} x}{\Gamma\left(Y_{1}, T, P\right)}},
\end{aligned}
$$

with $\left.[\beta]_{\Sigma}(T, P) \equiv \beta_{1}(T, P)\right|_{\Sigma}-\left.\beta_{2}(T, P)\right|_{\Sigma}$, the functions $\alpha, \beta$ and $\Gamma$ being respectively defined with (4), (17) and (21). Let us note that the system (27) is a mixed hyperbolic equation/ "parabolic" equation and that the system (28) is a non-homogeneous incompressible Navier-Stokes system. The pressure $P(t)$ is named thermodynamic pressure since it is directly related to the thermodynamic temperature $T(t, x)$ and to the density $\rho(t, x)$ through the equation of state $\rho\left(Y_{1}, T, P\right)$. At the opposite, the pressure $\Pi(t, x)$ is named dynamic pressure in reference to the pressure in the classical incompressible Navier-Stokes system.

Let us recall that, under the boundary conditions (8)-(9), the equation (30) is equivalent to the the Neumann compatibility condition

$$
\int_{\Omega} G(t, x) \mathrm{d} x=0 .
$$

It is important to note that any change in (8)-(9) will modify the equation (30)(b). For example, if we replace the boundary condition (9)(b) with the non-homogeneous Neumann condition $\left.\lambda \nabla T(t, x) \cdot n(x)\right|_{\partial \Omega}=q_{0}(t, x)$ 
where $q_{0}(t, x)$ is a given function, the equation (30)(b) will be given by

$$
P^{\prime}(t)=\widehat{P}^{\prime}(t)+\frac{\int_{\partial \Omega} \beta\left(Y_{1}, T, P\right) q_{0}(t, x) \cdot n(x) \mathrm{d} s}{\int_{\Omega} \frac{\mathrm{d} x}{\Gamma\left(Y_{1}, T, P\right)}},
$$

$\widehat{P}^{\prime}(t)$ being given by the right hand side of $(30)(\mathrm{b})$.

\subsection{A remark on the filtering out of the acoustic waves}

Because of the hyperbolic part of the diphasic Navier-Stokes system (1), the variations of the quantities $T, P, \rho$ and $u$ move with the fluid particles at the material velocity $u(t, x)$ but also with the acoustic waves at the velocity $|u(t, x) \pm c(t, x)|$ (in the monodimensional case). The asymptotic expansion supposes that $c(t, x) \gg|u(t, x)|$ at any point $(t, x)$ (cf. the low Mach number Hypothesis 2.6). Thus, the informations moving with the acoustic waves - i.e. the thermodynamic pressure waves - move at a celerity which is equal to the infinity. This changes the nature of the system which becomes elliptic via the constraint (28)(a) and the new variable $\Pi(t, x)$ in $(28)$ (b). We recover this elliptic charactere in the pressure equation (30)(b) since any change in the topology of the interface $\Sigma(t)$ modifies instantaneously the average thermodynamic pressure $P$ at any point $x$ of $\Omega$. Another important consequence of this elliptic charactere is that the DLMN system gives only an average thermodynamic pressure $P(t)$ in $\Omega$ whose characteristic time of variation is infinitely bigger than the characteristic time of acoustic waves in (1). This means that the DLMN system filters out any variations of the quantities $T, P, \rho$ and $u$ at time and space scales of the acoustic waves.

\section{BASIC PROPERTIES}

The DLMN system (27)-(30) - with the boundary conditions (8)-(9) - has conservation properties. Moreover, the DLMN system allows to recover, with minimal thermodynamic hypothesis, some natural physical results concerning the dilation and the compression of bubbles, and it degenerates toward the incompressible NavierStokes system in one of the two fluids - let us say in the fluid 2 - if the compressibility of the fluid 2 is close to zero compared to the compressibility of the fluid 1 (a liquid versus a gas for example). At last, it is possible to show that the total entropy of the mixture in $\Omega$ decreases and is minimized by a finite quantity.

\subsection{Conservation properties}

The DLMN system verifies the following mass and energy conservation properties:

Lemma 3.1. The DLMN system verifies

$$
\left\{\begin{array}{l}
\frac{\mathrm{d}}{\mathrm{d} t} \int_{\Omega_{k}(t)} \rho_{k}(t, x) \mathrm{d} x=0 \quad \text { for } \quad k \in\{1,2\}, \quad \text { (a) } \\
\frac{\mathrm{d}}{\mathrm{d} t} \int_{\Omega} \rho(t, x) \varepsilon(t, x) \mathrm{d} x=0 .
\end{array}\right.
$$

Under the boundary conditions (8)(a) and (9)(a), the relation (32)(a) is trivial since the divergence constraint (28)(a) is equivalent to the classical continuity equation. The relation (32)(b) is deduced from the temperature equation $(27)(\mathrm{b})$ which is equivalent to the energy equation $\rho D_{t} \varepsilon=-P \cdot G+\nabla \cdot(\lambda \nabla T)$. Thus, we conclude by noting that the pressure $P$ does not depend on the space variable $x$, by noting that the function $G(t, x)$ verifies the Neumann compatibility condition (31) and by using the boundary condition (9)(b).

Let us remark that the DLMN system neglects the kinetic energy $u^{2} / 2$ in the energy conservation equation (32)(b): this is coherent with the hypothesis of a low Mach number flow ( $f f$. Hypothesis 2.6) since $\mathcal{O}$ (kinetic energy $)=M_{*}^{2} \times \mathcal{O}$ (internal energy) where $M_{*}$ is the Mach number of the flow. 


\subsection{Dilation and compression of a bubble}

Another trivial property is the one which concerns the dilation or the compression of a bubble of fluid 1:

Lemma 3.2. The variation of the volume $\operatorname{Vol}\left(\Omega_{1}\right)$ of the domain $\Omega_{1}$ is given by

$$
\frac{\mathrm{d}}{\mathrm{d} t} \operatorname{Vol}\left(\Omega_{1}(t)\right)=\frac{1}{P(t)} \int_{\Sigma(t)} \bar{\beta}\left(\Omega_{1}, T, P\right) \lambda \nabla T \cdot n_{1 \rightarrow 2} \mathrm{~d} \Sigma+\frac{\mathcal{G}_{1}\left(\Omega_{1}, T, P\right)}{P(t)}
$$

where

$$
\left\{\begin{array}{l}
\bar{\beta}\left(\Omega_{1}, T, P\right)=\frac{\beta_{1}(T, P)}{\bar{\Gamma}_{2}\left(\Omega_{2}, T, P\right)}+\frac{\beta_{2}(T, P)}{\bar{\Gamma}_{1}\left(\Omega_{1}, T, P\right)} \\
\mathcal{G}_{1}\left(\Omega_{1}, T, P\right)=\int_{\Omega_{2}(t)} \frac{\frac{\partial \beta_{2}}{\partial T}(T, P)}{\bar{\Gamma}_{1}\left(\Omega_{1}, T, P\right)} \lambda_{2}(\nabla T)^{2} \mathrm{~d} x-\int_{\Omega_{1}(t)} \frac{\frac{\partial \beta_{1}}{\partial T}(T, P)}{\bar{\Gamma}_{2}\left(\Omega_{2}, T, P\right)} \lambda_{1}(\nabla T)^{2} \mathrm{~d} x, \\
\bar{\Gamma}_{k}\left(\Omega_{k}, T, P\right)=\frac{\int_{\Omega} \frac{\mathrm{d} x}{\int_{\Omega_{k}(t)} \frac{\mathrm{d} x}{\Gamma_{k}(T, P)}}}{}
\end{array}\right.
$$

Thus, when the equations of state are such that $\frac{\partial \beta_{k}}{\partial T}(T, P)=0$, we obtain

$$
\frac{\mathrm{d}}{\mathrm{d} t} \operatorname{Vol}\left(\Omega_{1}(t)\right)=\frac{1}{P(t)} \int_{\Sigma(t)} \bar{\beta}\left(\Omega_{1}, T, P\right) \lambda \nabla T \cdot n_{1 \rightarrow 2} \mathrm{~d} \Sigma .
$$

This lemma allows us to deduce the following corollary:

Corollary 3.1. When the equations of state are such that

$$
\forall(T, P): \quad \beta_{k}(T, P)>0 \quad \text { and } \quad \frac{\partial \beta_{k}}{\partial T}(T, P)=0,
$$

the deformation of the domain $\Omega_{1}(t)$ is such that

$$
\left\{\begin{array}{l}
\text { If }\left.\nabla T \cdot n_{1 \rightarrow 2}\right|_{\Sigma(t)}>0 \text { (i.e. "cold bubbles" of fluid 1), there is dilation of } \Omega_{1}(t) \text { and compression of } \Omega_{2}(t) ; \\
\text { If }\left.\nabla T \cdot n_{1 \rightarrow 2}\right|_{\Sigma(t)}<0 \text { (i.e. "hot bubbles" of fluid 1), there is compression of } \Omega_{1}(t) \text { and dilation of } \Omega_{2}(t) .
\end{array}\right.
$$

Let us note that when (36) is not verified, we can say nothing about dilation nor compression of $\Omega_{k}(t)$. For example, there will be "competition" between the two fluids when $\beta_{1}(T, P)>0$ and $\beta_{2}(T, P)<0$. Nevertheless, the hypothesis (36) is verified for a large class of equations of state used to model gases and liquids. Indeed, it is possible to show that, under the thermodynamic Hypothesis 2.1, the thermodynamic function $\beta_{k}(T, P)$ defined with $(17)$ is also given by $\beta_{k}(T, P)=\frac{\mathcal{G}_{k}}{\Gamma_{k}}(T, P)$ knowing that $\mathcal{G}_{k} \equiv \frac{1}{\rho_{k}} \cdot \frac{\partial P_{k}}{\partial \varepsilon_{k}}\left(\rho_{k}, \varepsilon_{k}\right)$ is the Grüneisen function of the fluid $k$ whose the equation of state is defined with $P_{k}\left(\rho_{k}, \varepsilon_{k}\right)$ (the function $P_{k}$ being the thermodynamic pressure of the fluid $k$ ). The Grüneisen function $\mathcal{G}_{k}$ is an important thermodynamic function for general equations of state (see [27] for example). We deduce from that equation of state that the relations (36) are verified if and only if the Grüneisen function $\mathcal{G}_{k}$ verifies

$$
\exists \xi_{k}(P)>0 \quad \text { such that } \mathcal{G}_{k}(T, P)=\xi_{k}(P) \cdot \Gamma_{k}(T, P)
$$

(the function $\Gamma_{k}$ defined with (21) is strictly positive when the thermodynamic Hypothesis 3.2 is satisfied). Of course, we obtain in that case that $\beta_{k}(T, P)=\beta_{k}(P)=\xi_{k}(P)$. Let us now consider the important case of 
generalized van der Waals type fluids. A generalized van der Waals equation of state $P\left(\rho_{k}, \varepsilon_{k}\right)$ for the fluid $k$ is defined through the formula

$$
P\left(\rho_{k}, \varepsilon_{k}\right)=\frac{\gamma_{k}-1}{1-b_{k} \rho_{k}}\left(\rho_{k} \varepsilon_{k}-\pi_{k}+a_{k} \rho_{k}^{2}\right)-\left(\pi_{k}+a_{k} \rho_{k}^{2}\right)
$$

where $a_{k}, b_{k}, \pi_{k}$ are positive constants and where $\gamma_{k}>1$ is another positive constant (see $[3,16,27]$ for example). And, we verify that the equation of state (38) satisfies (37) if and only if $a_{k}=0$; in that case, we find

$$
\beta_{k}(T, P)=\beta_{k}(P)=\frac{\gamma_{k}-1}{\gamma_{k}} \cdot \frac{P}{P+\pi_{k}} .
$$

The generalized van der Waals model with $a_{k}=0$ is important since it is classical to model the vapor phase by taking $a_{k}=b_{k}=\pi_{k}=0$ (which corresponds to a perfect gas type modelling) and since it is classical to model the liquid phase by taking $a_{k}=0$ and $\pi_{k}>0$ (which corresponds to a stiffened gas type modelling when $b_{k}=0$ and to a covolume gas type modelling when $\left.b_{k}>0\right)$.

Proof of lemma 3.2. Due to the divergence constraint (28)(a), the variation of the volume $\operatorname{Vol}\left(\Omega_{k}\right)$ is given by $\frac{\mathrm{d}}{\mathrm{d} t} \operatorname{Vol}\left(\Omega_{k}(t)\right)=\int_{\Omega_{k}(t)} G(t, x) \mathrm{d} x$. Thus, we obtain the result by integrating (29) on the domain $\Omega_{1}(t)$ and by taking into account (30).

\subsection{Degenerescence of the DLMN system}

Let us suppose that the fluids 1 and 2 are respectively "close" to a perfect gas and to a classical liquid. Moreover, we suppose that the thermal conductivity of the two fluids are "similar". We sum up these physical hypothesis with:

Hypothesis 3.1. The equations of state of fluids 1 and 2 are such that

$$
\mathcal{O}\left[\beta_{1}(T, P)\right]=1 \quad \text { and } \quad \mathcal{O}\left[\Gamma_{1}(T, P)\right]=1
$$

and such that

$$
\left\{\begin{array}{l}
\mathcal{O}\left[\beta_{2}(T, P)\right]=0 \\
\mathcal{O}\left[\Gamma_{2}(T, P)\right] \gg 1 \\
\mathcal{O}\left[\lambda_{2}(T, P)\right]=\mathcal{O}\left[\lambda_{1}(T, P)\right]
\end{array}\right.
$$

The relations (40) mean that the fluid 1 is "close" to a perfect gas. The relation (41)(a) means that the fluid 2 is infinitely more incompressible than the fluid 1 ; the relation $(41)(\mathrm{b})$ means that the product $\rho_{2} c_{2}^{2}(T, P)$ is very high which implies for example that the density of the fluid 2 is very high and that the sound velocities of the fluids 1 and 2 are similar; at last, the relation (41)(c) means that the conductivity of the fluid 2 does not have to be very high. In that physical situation, we can say that the fluid 2 is "close" to a classical liquid. Let us note that when each equation of state $P\left(\rho_{k}, \varepsilon_{k}\right)$ is of the generalized van der Waals type (38) with $a_{k}=0$, we have already remark that $\beta_{k}(T, P)$ is given by (39); we could also verify that $\Gamma_{k}(T, P)=\frac{\gamma_{k}}{1-b_{k} \rho_{k}(T, P)} \cdot \frac{P+\pi_{k}}{P}$. These two formulae show that the Hypothesis 3.1 is verified as soon as $\pi_{1}=\mathcal{O}(1), P=\mathcal{O}(1), \pi_{2} \gg \pi_{1}$ (with for example $b_{k}=0$ and $\gamma_{k}=5 / 3$ ). This explains why (38) with $\pi_{2} \gg \pi_{1}$ is often used to model a liquid phase. This also indicates that when the thermodynamic pressure is high in the sense that $\mathcal{O}(P)=\pi_{2}$, the liquid phase may not be seen as an incompressible phase.

We can now write:

Lemma 3.3. Under the Hypothesis 3.1, the DLMN system degenerates (formally) to the incompressible NavierStokes system in the domain $\Omega_{2}(t)$, the temperature equation (27)(b) being in $\Omega_{2}(t)$ a simple parabolic equation (i.e. without any source term). Moreover, the volumes of $\Omega_{1}(t)$ and $\Omega_{2}(t)$ do not change with time. 
This lemma is a consequence of the nullity of the velocity divergence in $\Omega_{2}(t)$ since the function $\left.G(t)\right|_{\Omega_{2}(t)}$ defined with (29) is formally equal to zero under the Hypothesis 3.1. It is important to note that, although the total volume of $\Omega_{1}(t)$ does not change, the volume of each bubble of fluid 1 can change. More precisely, any variation of the volume of a bubble of fluid 1 modifies instantaneously the volume of the other bubbles of fluid 1. This is a consequence of the elliptic charactere of the DLMN system: $c f$. Section 2.4. Thus, we can say that the fluid 1 is globally incompressible but locally compressible when the fluid 2 is incompressible. Let us remark that this lemma may not be verified when the thermal conductivity of fluid 2 is very high since, in that situation, the Fourier term in (29) may not be neglected into the domain $\Omega_{2}(t)$.

Moreover, we can deduce the following corollary from Lemma 3.3:

Corollary 3.2. Under the Hypothesis 3.1, when the domains $\Omega_{k}$ are such that $\mathcal{O}\left[\operatorname{Vol}\left(\Omega_{2}\right) / \operatorname{Vol}\left(\Omega_{1}\right)\right] \ll \min \left[\mathcal{O}\left(\Gamma_{2}\right), \mathcal{O}\left(\beta_{2}^{-1}\right)\right]$ and when the equations of state of the fluid 1 are such that

$$
\forall(T, P): \quad \beta_{1}(T, P)>0 \quad \text { and } \quad \frac{\partial \beta_{1}}{\partial T}(T, P)=0,
$$

the pressure equation (30) can be approximated with

$$
P^{\prime}(t)=\frac{\int_{\Sigma(t)} \beta_{1}(T, P) \lambda \nabla T \cdot n_{1 \rightarrow 2} \mathrm{~d} \Sigma}{\int_{\Omega_{1}(t)} \frac{\mathrm{d} x}{\Gamma_{1}\left(Y_{1}, T, P\right)}}
$$

which implies that

$$
\left\{\begin{array}{l}
\text { If }\left.\nabla T \cdot n_{1 \rightarrow 2}\right|_{\Sigma(t)}>0 \text { (i.e. "cold bubbles" of fluid 1), the pressure } P(t) \text { increases; } \\
\text { If }\left.\nabla T \cdot n_{1 \rightarrow 2}\right|_{\Sigma(t)}<0 \text { (i.e. "hot bubbles" of fluid 1), the pressure } P(t) \text { decreases. }
\end{array}\right.
$$

and that

- the thermodynamic pressure $P(t)$ in $\Omega$ is only a function of the thermodynamic quantities in the fluid $1 ;$

- the more the volume of a bubble of fluid 1 is important, the more this bubble imposes the pressure $P(t)$ to the rest of the domain $\Omega^{1}$.

Let us remark that the condition $\mathcal{O}\left[\operatorname{Vol}\left(\Omega_{2}\right) / \operatorname{Vol}\left(\Omega_{1}\right)\right] \ll \min \left[\mathcal{O}\left(\Gamma_{2}\right), \mathcal{O}\left(\beta_{2}^{-1}\right)\right]$ means that when the volume of $\Omega_{1}(t)$ is tiny, the equation (43) may not be valid.

\subsection{Decreasing of the entropy and existence of a minimizer}

Let us recall that we suppose that each fluid verifies ( $c f$. Hypothesis 2.1):

Hypothesis 3.2. The equations of state $\rho_{k}(T, P) \equiv 1 / \tau_{k}(T, P)$ and $\varepsilon_{k}(T, P)$ of each fluid $k \in\{1,2\}$ are such that it exists a function $s_{k}\left(\tau_{k}, \varepsilon_{k}\right)$ verifying

$$
\left\{\begin{array}{l}
s_{k}\left(\tau_{k}, \varepsilon_{k}\right) \text { is a strictly convex function, } \\
-T \mathrm{~d} s_{k}=\mathrm{d} \varepsilon_{k}+P \mathrm{~d} \tau_{k}
\end{array}\right.
$$

\footnotetext{
${ }^{1}$ This kind of situation is numerically observed in [14] p. 645: "It then appears that in the closed system considered here, the bigger bubble imposes its pressure in the liquid".
} 
The function $s_{k}\left(\tau_{k}, \varepsilon_{k}\right)$ is the classical thermodynamic entropy of the fluid $k$. Due to the thermodynamic Hypothesis 3.2, we can write:

Lemma 3.4. The total entropy $\mathcal{S}(T, P)$ defined with

$$
\mathcal{S}(T, P)(t)=\int_{\Omega} \rho\left(Y_{1}, T, P\right) s\left(Y_{1}, T, P\right) \mathrm{d} x
$$

where $s\left(Y_{1}, T, P\right)=Y_{1} s_{1}(T, P)+\left(1-Y_{1}\right) s_{2}(T, P)$ verifies $\frac{\mathrm{d}}{\mathrm{d} t} \mathcal{S}(T, P)(t) \leq 0$. And, any equilibrium characterized by the equilibrium interface $\Sigma^{\infty}$ is a solution of the minimization problem

$$
\mathcal{S}\left(T^{\infty}, P^{\infty}\right)=\min _{T(x), P} \mathcal{S}(T, P)
$$

under the constraints

$$
\left\{\begin{array}{l}
\int_{\Omega_{k}} \rho_{k}[T(x), P] \mathrm{d} x=\mathcal{M}_{k}>0 \quad \text { for } \quad k \in\{1,2\}, \\
\sum_{k} \int_{\Omega_{k}} \rho_{k}[T(x), P] \varepsilon_{k}[T(x), P] \mathrm{d} x=\mathcal{E}, \\
T(x)>0 \quad \text { and } P>0, \\
\Omega_{k} \quad \text { fixed such that } \partial \Omega_{1}=\Sigma^{\infty},
\end{array}\right.
$$

$\mathcal{M}_{k}$ and $\mathcal{E}$ being strictly positive constants defined by the initial conditions. Moreover, the equilibrium $\left(T^{\infty}(x), P^{\infty}\right)$ is unique and $T^{\infty}(x)$ is a strictly positive constant $T^{\infty}$.

The proof of this lemma is classical: it uses the strict positivity of each Hessian matrix $\frac{\partial^{2} s_{k}}{\partial \tau_{k} \partial \varepsilon_{k}}\left(\tau_{k}, \varepsilon_{k}\right)-$ which is a direct consequence of $(44)(\mathrm{a})$ (see [6] for example) -, the differential relation (44)(b) and the strict positivity of the thermal conductivity $\lambda\left(Y_{1}, T, P\right)$. In Section 5 numerical results will clearly show that when at least one of the entropy $s_{k}\left(\tau_{k}, \varepsilon_{k}\right)$ is not a strictly convex function, the strict positivity of $\lambda\left(Y_{1}, T, P\right)$ is not a sufficient condition to obtain an asymptotic convergence of the DLMN system, the system becoming ill-posed.

From Lemma 3.4, we would like to prove that there is convergence to an unique and stable equilibrium when the time $t$ goes to infinity. Unfortunately, when the space dimension is greater or equal to two, the equilibrium interface $\Sigma^{\infty}$ is never unique for a given initial condition and the Lemma 3.4 is not sufficient to conclude. Nevertheless, in monodimensional geometry, the domain $\Omega_{k}(t>0)$ is always homeomorphic to the domain $\Omega_{k}(t=0)$ for any $k \in\{1,2\}$ (i.e. any fragmentation or coalescence is not possible when the space dimension is equal to one), and it is possible to obtain a better result (see the following section). The price to pay is that a monodimensional bubble is a very poor description of the reality!

\section{The DLMN SYSTEM FOR PERFECT GASES IN LAGRANGIAN FORMULATION}

We now focus on the case of two perfect gases which means that the equations of state are defined with

$$
\left\{\begin{array}{l}
\rho_{k}(T, P)=\frac{P}{R_{k} T} \\
\varepsilon_{k}(T, P)=\varepsilon_{k}(T)=\frac{R_{k} T}{\gamma_{k}-1}
\end{array}\right.
$$


where $R_{k}>0$ and $\gamma_{k}>1$. In that case, we obtain

$$
\left\{\begin{array}{l}
C_{p, k}(T, P)=C_{p, k}=\frac{\gamma_{k} R_{k}}{\gamma_{k}-1}, \\
\alpha_{k}(T, P)=\alpha_{k}(T)=\frac{1}{T} \\
\beta_{k}(T, P)=\beta_{k}=\frac{\gamma_{k}-1}{\gamma_{k}} \\
c_{k}(T, P)=c_{k}(T)=\sqrt{\gamma_{k} R_{k} T} \\
\Gamma_{k}(T, P)=\Gamma_{k}=\gamma_{k}
\end{array}\right.
$$

Let us note that lemma 3.1 is now given by

Lemma 4.1. The DLMN system written for two perfects gases verifies

$$
\left\{\begin{array}{l}
\frac{\mathrm{d}}{\mathrm{d} t}\left(P(t) \int_{\Omega_{k}(t)} \frac{\mathrm{d} x}{R_{k} T(t, x)}\right)=0 \text { for } k \in\{1,2\} \\
\frac{\mathrm{d}}{\mathrm{d} t}\left(P(t) \int_{\Omega} \frac{\mathrm{d} x}{\gamma\left(Y_{1}\right)-1}\right)=0
\end{array}\right.
$$

By using the energy conservation equation (50)(b), we obtain the corollary:

Corollary 4.1. When $0<P(t=0)<+\infty$, the DLMN system written for two perfects gases verifies

$$
\inf _{[0, \mathcal{T}[} P(t)>0 \quad \text { and } \quad \sup _{[0, \mathcal{T}[} P(t)<+\infty
$$

where $\mathcal{T} \in[0,+\infty]$ is the life time of the solution.

When the space is monodimensional, it is possible to write the DLMN system (27)-(30) in Lagrangian coordinates. This formulation is interesting since the interface $\Sigma(t)$ is fixed in Lagrangian coordinates. Moreover, when the equations of state are given by (48), it is possible to introduce a new variable - namely the entropic variable $\Psi$ : see Section 4.2 - which makes equivalent the temperature equation with a non-linear heat equation. This property will allow us to be confident concerning the asymptotic stability of the DLMN system in the case of two non-miscible perfect gases and will allow us to build an entropic monodimensional scheme in Section 5.

\subsection{Lagrangian formulation of the DLMN system}

When the geometry is monodimensional, it is possible to write the DLMN system in the time-mass variable $(\bar{t}, m)$ - i.e. in Lagrangian coordinates - by using the differential transformation

$$
\partial_{\bar{t}}=\partial_{t}+u(t, x) \partial_{x} \quad \text { and } \quad \partial_{m}=\rho^{-1} \partial_{x}
$$

In that Lagrangian space, we suppose that the topology of the flow is defined with

$$
\left.\Omega=\Omega_{1} \cup \Omega_{2} \cup \Sigma=\right]-L, L[\quad(L>0)
$$

and with

$$
\left.\Sigma=\left\{\Sigma^{a}, \Sigma^{b}\right\}, \quad \Omega_{1}=\right] \Sigma^{a}, \Sigma^{b}\left[, \quad \Omega_{2}=\Omega_{2}^{a} \cup \Omega_{2}^{b}, \quad \Omega_{2}^{a}=\right]-L, \Sigma^{a}\left[\quad \text { and } \quad \Omega_{2}^{b}=\right] \Sigma^{b}, L[
$$


(with of course $\left.-L<\Sigma^{a}<\Sigma^{b}<L\right)$. We note $\Sigma_{k}=\left\{\Sigma_{k}^{a}, \Sigma_{k}^{b}\right\}(k \in\{1,2\})$ the side of $\Sigma$ which is "seen" by the fluid $k$ (here, the fluid 1 is on the right of $\Sigma^{a}$ and on the left of $\Sigma^{b}$ ). By noting $\bar{t}$ with $t$, the DLMN system in Lagrangian coordinates is defined with

$$
\left\{\begin{array}{l}
\partial_{t} Y_{1}=0 \\
C_{p}(m) \partial_{t} T=R(m) T \frac{P^{\prime}(t)}{P(t)}+\partial_{m}\left[\eta(m, T, P) \partial_{m} T\right]
\end{array}\right.
$$

(the quantity $\eta$ is the thermal conductivity in the Lagrangian space and is equal to $\rho \lambda$ ), and with

$$
\begin{cases}\partial_{m} u=\bar{G}(t, m), & \text { (a) } \\ \partial_{t} u=-\partial_{m} \Pi, & \text { (b) }\end{cases}
$$

knowing that

$$
\bar{G}(t, m)=\frac{G}{\rho}=-\frac{R(m) T(t, m)}{\gamma(m)} \cdot \frac{P^{\prime}(t)}{P(t)^{2}}+\frac{\gamma(m)-1}{\gamma(m) P(t)} \partial_{m}\left[\eta(m, T, P) \partial_{m} T\right],
$$

this function verifying the Neumann compatibility condition

$$
\int_{-L}^{L} \bar{G}(t, m) \mathrm{d} m=0
$$

In (54)(b), we omit the gravity and the viscosity since these quantities are not relevant in monodimensional geometry. Since $Y_{1}(t, m)=Y_{1}(t=0, m)$, the topology (51)-(52) does not change with time which means that the interface $\Sigma(t>0)$ is always equal to $\Sigma(t=0) \equiv\left\{\Sigma^{a}, \Sigma^{b}\right\}$ in Lagrangian coordinates. This induces that the physical coefficient $\xi \in\left\{\gamma, R, C_{p}\right\}$ is defined with $\xi(m)=1_{\Omega_{1}}(m) \cdot \xi_{1}+\left(1-1_{\Omega_{1}}(m)\right) \cdot \xi_{2}$ and that the conductivity $\eta(m, T, P)$ is defined with $\eta(m, T, P)=1_{\Omega_{1}}(m) \cdot \eta_{1}(T, P)+\left(1-1_{\Omega_{1}}(m)\right) \cdot \eta_{2}(T, P)$ where $\eta_{k}=\lambda_{k} \cdot \frac{P}{R_{k} T}$. Moreover, due to the topology (51)-(52), the pressure equation is given with

$$
P^{\prime}(t)=\beta_{2}^{1} \cdot P(t) \frac{\left[\eta \partial_{m} T\right]_{\Sigma^{b}}-\left[\eta \partial_{m} T\right]_{\Sigma^{a}}}{\frac{R_{1}}{\gamma_{1}} \int_{\Sigma^{a}}^{\Sigma^{b}} T(t, m) \mathrm{d} m+\frac{R_{2}}{\gamma_{2}}\left(\int_{-L}^{\Sigma^{a}} T(t, m) \mathrm{d} m+\int_{\Sigma^{b}}^{L} T(t, m) \mathrm{d} m\right)}
$$

where $\beta_{2}^{1} \equiv \frac{\gamma_{1}-1}{\gamma_{1}}-\frac{\gamma_{2}-1}{\gamma_{2}}=\frac{\gamma_{1}-\gamma_{2}}{\gamma_{1} \gamma_{2}}$. Of course, the boundary conditions (8)-(9) are now defined with

$$
\begin{cases}\left.T\right|_{\Sigma_{1}^{a}}=\left.T\right|_{\Sigma_{2}^{a}}, & \text { (a) } \\ \left.\eta_{1} \partial_{m} T\right|_{\Sigma_{1}^{a}}=\left.\left.\eta_{2} \partial_{m} T\right|_{\Sigma_{2}^{a}} \equiv \eta \partial_{m} T\right|_{\Sigma_{2}^{a}}, & \text { (b) } \\ \left.T\right|_{\Sigma_{1}^{b}}=\left.T\right|_{\Sigma_{2}^{b}}, & \text { (a') } \\ \left.\eta_{1} \partial_{m} T\right|_{\Sigma_{1}^{b}}=\left.\left.\eta_{2} \partial_{m} T\right|_{\Sigma_{2}^{b}} \equiv \eta \partial_{m} T\right|_{\Sigma^{b}}, & \text { (b') } \\ \left.\partial_{m} T\right|_{ \pm L}=0, & \text { (c) } \\ \left.u\right|_{ \pm L}=0 & \text { (d) }\end{cases}
$$

A good property of the DLMN system written in Lagrangian coordinates is that the temperature equation (53)(b) and the pressure equation (57) are completely decoupled from the system (54). A direct consequence 
is that the velocity $u(t, m)$ can be defined with

$$
u(t, m)=\int_{-L}^{m} \bar{G}(t, m) \mathrm{d} m=\mathcal{U}[T(t, m), P(t)]
$$

where $\mathcal{U}$ is an ad hoc function which only depends on $T$ and $P$. Nevertheless, the coupling between the two equations (53)(b) and (57) through the source term $R T P^{\prime} / P$ complicates the analysis. By using a simple change of variable, we now show that it is possible to transform the temperature equation (53)(b) on a non-linear heat equation.

\subsection{Entropic Lagrangian formulation of the DLMN system}

By introducing the new variable

$$
\Psi(t, m) \equiv \frac{T(t, m)}{P(t)^{\frac{\gamma(m)-1}{\gamma(m)}}}
$$

it is not difficult to verify that the equations (53)-(58) now read

$$
\left\{\begin{array}{l}
\partial_{t} Y_{1}=0 \\
C_{p}(m) \partial_{t} \Psi=\partial_{m}\left[\eta(m, T, P) \partial_{m} \Psi\right]
\end{array}\right.
$$

and

with

$$
\left\{\begin{array}{l}
\partial_{m} u=\bar{G}(t, m), \quad \text { (a) } \\
\partial_{t} u=-\partial_{m} \Pi \quad \text { (b) }
\end{array}\right.
$$

$$
\bar{G}(t, m)=-\frac{R(m) \Psi(t, m)}{\gamma(m)} \cdot \frac{P^{\prime}(t)}{P(t)^{\frac{1+\gamma(m)}{\gamma(m)}}}+\frac{\gamma(m)-1}{\gamma(m) P(t)} \partial_{m}\left[P(t)^{\frac{\gamma(m)-1}{\gamma(m)}} \eta(m, T, P) \partial_{m} \Psi\right],
$$

where the pressure equation is given by

$$
P^{\prime}(t)=\beta_{2}^{1} \frac{\left[P^{\frac{\gamma-1}{\gamma}} \eta \partial_{m} \Psi\right]_{\Sigma^{b}}-\left[P^{\frac{\gamma-1}{\gamma}} \eta \partial_{m} \Psi\right]_{\Sigma^{a}}}{\int_{-L}^{L} \frac{R(m) \Psi(t, m)}{\gamma(m) P(t)^{\frac{1}{\gamma(m)}}} \mathrm{d} m}
$$

the boundary conditions being defined with

$$
\left\{\begin{array}{l}
\left.P(t)^{\frac{\gamma_{1}-1}{\gamma_{1}}} \Psi\right|_{\Sigma_{1}^{a}}=\left.P(t)^{\frac{\gamma_{2}-1}{\gamma_{2}}} \Psi\right|_{\Sigma_{2}^{a}}, \\
\left.P(t)^{\frac{\gamma_{1}-1}{\gamma_{1}}} \eta_{1} \partial_{m} \Psi\right|_{\Sigma_{1}^{a}}=\left.\left.P(t)^{\frac{\gamma_{2}-1}{\gamma_{2}}} \eta_{2} \partial_{m} \Psi\right|_{\Sigma_{2}^{a}} \equiv P^{\frac{\gamma-1}{\gamma}} \eta \partial_{m} \Psi\right|_{\Sigma^{a}}, \\
\left.P(t)^{\frac{\gamma_{1}-1}{\gamma_{1}}} \Psi\right|_{\Sigma_{1}^{b}}=\left.P(t)^{\frac{\gamma_{2}-1}{\gamma_{2}}} \Psi\right|_{\Sigma_{2}^{b}}, \\
\left.P(t)^{\frac{\gamma_{1}-1}{\gamma_{1}}} \eta_{1} \partial_{m} \Psi\right|_{\Sigma_{1}^{b}}=\left.\left.P(t)^{\frac{\gamma_{2}-1}{\gamma_{2}}} \eta_{2} \partial_{m} \Psi\right|_{\Sigma_{2}^{b}} \equiv P^{\frac{\gamma-1}{\gamma}} \eta \partial_{m} \Psi\right|_{\Sigma^{b}}, \\
\left.\partial_{m} \Psi\right|_{ \pm L}=0 \\
\left.u\right|_{ \pm L}=0
\end{array}\right.
$$


Let us note that the lemma 4.1 is now given by:

Lemma 4.2. The DLMN system written for two perfects gases in Lagrangian coordinates and in function of the variable $\Psi$ verifies

$$
\begin{cases}\frac{\mathrm{d}}{\mathrm{d} t} \mathcal{E}(t)=0 \quad \text { where } \quad \mathcal{E}(t)=\int_{-L}^{L} \frac{R(m) P(t)^{\frac{\gamma(m)-1}{\gamma(m)}} \Psi(t, m)}{\gamma(m)-1} \mathrm{~d} m \\ \frac{\mathrm{d}}{\mathrm{d} t} \mathcal{L}(t)=0 \quad \text { where } \quad \mathcal{L}(t)=\int_{-L}^{L} \frac{R(m) \Psi(t, m)}{P(t)^{\frac{1}{\gamma(m)}} \mathrm{d} m}\end{cases}
$$

The relation $(65)(\mathrm{b})$ corresponds to the relation $\frac{\mathrm{d}}{\mathrm{d} t} \int_{-L}^{L} \frac{\mathrm{d} m}{\rho}=0$ i.e. to the conservation of the physical volume of $\Omega$. The main advantage of this new formulation is that the temperature equation (53)(b) is replaced by the more simple parabolic equation $(60)(\mathrm{b})$. Nevertheless, the boundary conditions (64) on the interface $\Sigma$ are more complicated since they directly depend on the pressure $P(t)$. Let us emphasize that, since the quantity

$$
s(\Psi)(t, m)=-C_{p}(m) \log \Psi(t, m)
$$

is the thermodynamic entropy for a perfect gas when $\Psi=T / P^{\frac{\gamma-1}{\gamma}}$, Lemma 3.4 shows that the function $\mathcal{S}(\Psi)(t) \equiv \int_{\Omega} s(\Psi)(t, m) \mathrm{d} m$ is a decreasing function - which is a classical result for an heat equation of the type $(60)(\mathrm{b})$ - and that the equilibrium

$$
\left\{\begin{array}{l}
\Psi^{\infty}(m)=T^{\infty} /\left(P^{\infty}\right)^{\frac{\gamma(m)-1}{\gamma(m)}} \quad \text { knowing that } \quad T^{\infty}=\frac{\mathcal{E}}{\int_{-L}^{L} \frac{R(m)}{\gamma(m)-1} \mathrm{~d} m} \\
P^{\infty}=\frac{\mathcal{E}}{\mathcal{L}} \cdot \frac{\int_{-L}^{L} R(m) \mathrm{d} m}{\int_{-L}^{L} \frac{R(m)}{\gamma(m)-1} \mathrm{~d} m}
\end{array}\right.
$$

is the unique solution of the minimization problem

$$
\mathcal{S}\left(\Psi^{\infty}\right)=\min _{\Psi(m), P} \mathcal{S}(\Psi)
$$

under the constraints

$$
\left\{\begin{array}{l}
\int_{-L}^{L} \frac{R(m) \Psi(m)}{P^{\frac{1}{\gamma(m)}}} \mathrm{d} m=\mathcal{L}>0 \\
\int_{-L}^{L} \frac{R(m) P^{\frac{\gamma(m)-1}{\gamma(m)}} \Psi(m)}{\gamma(m)-1} \mathrm{~d} m=\mathcal{E}>0 \\
\Psi(m)>0 \text { and } P>0
\end{array}\right.
$$

Thus, we name entropic variable the variable $\Psi$ and we name entropic Lagrangian formulation this last formulation of the DLMN system. We can note that the final topology of the flow in the physical space is given by $\Omega_{k}^{\infty}$ knowing that $\operatorname{Vol}\left(\Omega_{1}^{\infty}\right)=\int_{\Sigma^{a}}^{\Sigma^{b}} \mathrm{~d} m / \rho_{1}^{\infty}, \operatorname{Vol}\left(\Omega_{2}^{a} \infty\right)=\int_{-L}^{\Sigma^{a}} \mathrm{~d} m / \rho_{2}^{\infty}$ and $\operatorname{Vol}\left(\Omega_{2}^{b^{\infty}}\right)=\int_{\Sigma^{b}}^{L} \mathrm{~d} m / \rho_{2}^{\infty}$ where $\rho_{k}^{\infty}=P^{\infty} /\left(R_{k} T^{\infty}\right)$ (let us recall that the topology in the Lagrangian space is defined with $(51)-(52)$ ). 


\subsection{A remark on the asymptotic stability}

Since the pressure $P(t)$ remains bounded (see Cor. 4.1), the boundary conditions on $\Sigma(t)$ are a priori well defined. By admitting that the function $\Psi(t, m)$ remains a classical solution of the non-linear parabolic equation (60)(b) (this topic is beyond the scope of that paper) and using the decreasing of the entropy $\mathcal{S}(\Psi)(t)$, we "can" say that for any strictly positive initial conditions, $(\Psi(t, m), P(t))$ converges toward the equilibrium $\left(\Psi^{\infty}(m), P^{\infty}\right)$ which is the unique minimizer of $\mathcal{S}(\Psi)$, and that the topology $\Omega_{k}(t)$ in the physical space converges toward the unique topology $\Omega_{k}^{\infty}$ when $t$ goes to infinity. Moreover, we also obtain that the final equilibrium $\left(\Psi^{\infty}(m), P^{\infty}\right)$ - and then $\left(T^{\infty}, P^{\infty}, \Omega_{k}^{\infty}\right)$ - is a stable equilibrium with respect to the initial conditions since the final entropy $\mathcal{S}\left(\Psi^{\infty}\right)$ is a continuous function of $\left(T(t=0, x), P(t=0), \Omega_{k}(t=0)\right)$.

Of course, we obtain this heuristics because any fragmentation or coalescence is not possible in monodimensional geometry which allows to define an unique final topology $\Omega_{k}^{\infty}$. In two or three dimensions, the problem is much more difficult, especially for the question concerning the stability of the final topology $\Omega_{k}^{\infty}$ with respect to the initial topology $\Omega_{k}(t=0)$.

\section{AN ENTROPIC LAGRANGIAN SCHEME FOR TWO PERFECT GASES}

The time subscript, the mass subscript and the interface subscript are respectively noted $n, i$ and $i+1 / 2$. The number of meshes is equal to $i_{\max }$ and the mesh size $\Delta m$ is constant, the time step being equal to $\Delta t$. We suppose that the topology of the flow is given by (51)-(52) in Lagrangian coordinates. The mesh in $\Omega_{2}^{a}$ which has an interface equal to the interface $\Sigma^{a}$ is noted $I^{a}$; the mesh in $\Omega_{1}$ which has an interface equal to the interface $\Sigma^{b}$ is noted $I^{b}$ (thus, the subscript $I^{a}+1 / 2$ refers to $\Sigma^{a}$ and the subscript $I^{b}+1 / 2$ refers to $\Sigma^{b}$ ). The quantities $\rho, T$ and $Y_{1}$ are computed at the center of each mesh $i$; the velocity $u$ is computed at each interface $i+1 / 2$ : then, the proposed scheme will be a MAC scheme [13].

By using the entropic Lagrangian formulation (59)-(64) of the DLMN system, we propose the scheme

$$
\left\{\begin{aligned}
Y_{i}^{n+1} & =Y_{i}^{n} \\
\Psi_{i}^{n+1} & =\Psi_{i}^{n}+\frac{\Delta t}{C_{p, i}}[D \eta D \Psi]_{i}^{n} \\
P^{n+1} & =\wp\left(P^{n}, \Psi^{n}, \Psi^{n+1}\right) \\
u_{i+1 / 2}^{n} & =u_{i-1 / 2}^{n}+\Delta m \bar{G}_{i}^{n} \text { if } i \neq 0
\end{aligned}\right.
$$

where

$$
\bar{G}_{i}^{n}=-\frac{R_{i} \Psi_{i}^{n}}{\gamma_{i}} \cdot \frac{\mathcal{P}^{\prime}\left(P^{n}, \Psi^{n}\right)}{P^{n \frac{1+\gamma_{i}}{\gamma_{i}}}}+\frac{\gamma_{i}-1}{\gamma_{i} P^{n \frac{1}{\gamma_{i}}}} \cdot[D \eta D \Psi]_{i}^{n} .
$$

The discrete operator $[D \eta D \Psi]_{i}$ will be defined in Section 5.1. The quantity $\mathcal{P}^{\prime}\left(P^{n}, \Psi^{n}\right)$ is an estimation of $P^{\prime}\left(t^{n}\right)$ defined with (63): it will be explicited in Section 5.2. The operator $\wp\left(P^{n}, \Psi^{n}, \Psi^{n+1}\right)$ estimates the pressure $P^{n+1}$ at time $t^{n+1}$ and will be given in Section 5.3. At last, when we know $\Psi_{i}^{n+1}$ and $P^{n+1}$, we compute the temperature with $T_{i}^{n+1}=\Psi_{i}^{n+1} \cdot P^{n+1} \frac{\gamma_{i}-1}{\gamma_{i}}$ and the density with $\rho_{i}^{n+1}=P^{n+1} /\left(R_{i} T_{i}^{n+1}\right)$.

\subsection{The discrete operator $[D \eta D \Psi]_{i}$}

The discrete operator $[D \eta D \Psi]_{i}$ is defined with

$$
[D \eta D \Psi]_{i}=\frac{[\eta D \Psi]_{i+1 / 2}-[\eta D \Psi]_{i-1 / 2}}{\Delta m} .
$$

The definition of the flux $[\eta D \Psi]_{i+1 / 2}$ on each interface $i+1 / 2$ is linked to the boundary conditions (64). 
When the mesh $i$ has no interface belonging to $\partial \Omega \cap \Sigma$ : in that case, we use

$$
\text { if } i \notin\left\{0, I^{a}, I^{b}, i_{\max }\right\}:\left\{\begin{array}{l}
{[\eta D \Psi]_{i+1 / 2} \equiv \eta_{i+1 / 2} \cdot \frac{\Psi_{i+1}-\Psi_{i}}{\Delta m},} \\
\eta_{i+1 / 2} \in\left[\min \left(\eta_{i}, \eta_{i+1}\right), \max \left(\eta_{i}, \eta_{i+1}\right)\right] .
\end{array}\right.
$$

To define $\eta_{i+1 / 2}$, we may choose the formula $\eta_{i+1 / 2}=\frac{\eta_{i}+\eta_{i+1}}{2}$ or the formula $\eta_{i+1 / 2}=\frac{2 \eta_{i} \eta_{i+1}}{\eta_{i}+\eta_{i+1}}$.

When the mesh $i$ has an interface belonging to $\partial \Omega=\{-L, L\}$ : of course, we define the thermal flux with

$$
\text { if } i \in\left\{0, i_{\max }\right\}: \quad[\eta D \Psi]_{i+1 / 2} \equiv 0,
$$

which is a discretized version of $(64)(\mathrm{c})$.

When $i$ has an interface belonging to the interface $\Sigma=\left\{\Sigma^{a}, \Sigma^{b}\right\}$ : near the interface $\Sigma^{a}$, a natural choice is to use the formulae

$$
\text { if } i=I^{a}:\left\{\begin{array}{l}
{[\eta D \Psi]_{\Sigma_{1}^{a}}=\eta_{I^{a}+1 / 2} \cdot \frac{\Psi_{I^{a}+1}-P^{-\beta_{2}^{1}} \Psi_{I^{a}}}{\Delta m},} \\
{[\eta D \Psi]_{\Sigma_{2}^{a}}=\eta_{I^{a}+1 / 2} \cdot \frac{P^{\beta_{2}^{1}} \Psi_{I^{a}+1}-\Psi_{I^{a}}}{\Delta m}}
\end{array}\right.
$$

which respects the boundary condition $(64)(\mathrm{b})$. Let us recall that $\Sigma_{k}=\left\{\Sigma_{k}^{a}, \Sigma_{k}^{b}\right\}(k \in\{1,2\})$ is the side of $\Sigma=\left\{\Sigma^{a}, \Sigma^{b}\right\}$ "seen" by the fluid $k$ and that $\beta_{2}^{1}=\frac{\gamma_{1}-\gamma_{2}}{\gamma_{1} \gamma_{2}}$. Using (72), we deduce that when $i \in\left\{I^{a}, I^{a}+1\right\}$, the discrete operator $[D \eta D \Psi]_{i}$ is defined with

$$
\left\{\begin{array}{l}
{[D \eta D \Psi]_{I^{a}}=\frac{[\eta D \Psi]_{\Sigma_{2}^{a}}-[\eta D \Psi]_{I^{a}-1 / 2}}{\Delta m}} \\
{[D \eta D \Psi]_{I^{a}+1}=\frac{[\eta D \Psi]_{I^{a}+3 / 2}-[\eta D \Psi]_{\Sigma_{1}^{a}}}{\Delta m}}
\end{array}\right.
$$

By symmetry, we define in the same way the discrete operator $[D \eta D \Psi]_{i}$ near the interface $\Sigma^{b}$ i.e. when $i \in\left\{I^{b}, I^{b}+1\right\}$. The conductivity $\eta_{I^{a}+1 / 2}$ has to be now defined: it is not difficult to verify that the formulae (75)

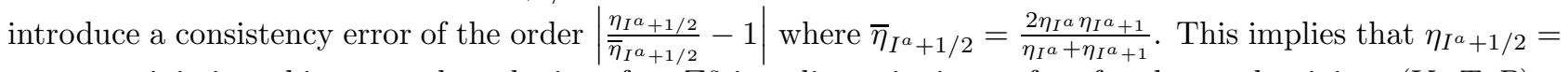
$\bar{\eta}_{I^{a}+1 / 2}$ minimizes this error when the interface $\Sigma^{a}$ is a discontinuity surface for the conductivity $\eta\left(Y_{1}, T, P\right)$.

\subsection{The discrete operator $\mathcal{P}^{\prime}(P, \Psi)$}

The discrete operator $\mathcal{P}^{\prime}(P, \Psi)$ used in $(71)$ is defined with

$$
\mathcal{P}^{\prime}(P, \Psi)=\beta_{2}^{1} \cdot \frac{\left[P^{\frac{\gamma-1}{\gamma}} \eta D \Psi\right]_{\Sigma^{b}}-\left[P^{\frac{\gamma-1}{\gamma}} \eta D \Psi\right]_{\Sigma^{a}}}{\sum_{i} \frac{R_{i} \Psi_{i}}{\gamma_{i} P^{\frac{1}{\gamma_{i}}}} \Delta m},
$$

which is an approximation of (63). This definition allows to easily obtain:

Lemma 5.1. The formulae (71) to (77) define a scheme which satisfies the discretized version $\sum_{i} \bar{G}_{i}^{n} \Delta m=0$ of the Neumann compatibility condition (56). A direct consequence is that the boundary condition (64)(d) is compatible with this scheme since $u_{1 / 2}^{n}=0$ implies that $u_{i_{\max }+1 / 2}^{n}=0$ when $u_{i+1 / 2}^{n}$ is computed with (70)(d). 


\subsection{The discrete operator $\wp\left(P^{n}, \Psi^{n}, \Psi^{n+1}\right)$}

There are three possibilities to define the discrete operator $\wp\left(P^{n}, \Psi^{n}, \Psi^{n+1}\right)$ giving the pressure $P^{n+1}$ in $(70)(c)$ : the two first possibilities define an implicit non-linear scheme since $\wp$ depends on $\Psi^{n+1}$, schemes which are partially conservative; the last possibility defines an explicit scheme which is not at all conservative.

\subsubsection{Conservative scheme in energy}

The relation (65)(a) allows to deduce the following formula for $\wp$ :

$$
\left\{\begin{array}{l}
P^{n+1} \equiv \wp\left(P^{n}, \Psi_{i}^{n}, \Psi_{i}^{n+1}\right)=X \quad \text { where } \quad X \quad \text { is the unique positive solution of } \\
x^{\zeta\left(\gamma_{1}\right)} \cdot \mathcal{A}_{1}\left(\Psi^{n+1}\right)+x^{\zeta\left(\gamma_{2}\right)} \cdot \mathcal{A}_{2}\left(\Psi^{n+1}\right)=\mathcal{B}\left(P^{n}, \Psi^{n}\right)
\end{array}\right.
$$

where

$$
\left\{\begin{array}{l}
\mathcal{A}_{1}(\Psi)=\frac{R_{1}}{\gamma_{1}-1} \cdot \sum_{i=I^{a}+1}^{I^{b}} \Psi_{i}, \\
\mathcal{A}_{2}(\Psi)=\frac{R_{2}}{\gamma_{2}-1} \cdot\left(\sum_{i=1}^{I^{a}} \Psi_{i}+\sum_{I^{b}+1}^{i_{\max }} \Psi_{i}\right), \\
\mathcal{B}(P, \Psi)=P^{\zeta\left(\gamma_{1}\right)} \cdot \mathcal{A}_{1}(\Psi)+P^{\zeta\left(\gamma_{2}\right)} \cdot \mathcal{A}_{2}(\Psi), \\
\zeta(\gamma)=\frac{\gamma-1}{\gamma} .
\end{array}\right.
$$

By construction, this operator $\wp$ allows to obtain a conservative scheme in energy. Nevertheless, this scheme is not conservative in volume. Let us remark that the study of the function $x \mapsto x^{\zeta\left(\gamma_{1}\right)} \cdot \mathcal{A}_{1}\left(\Psi^{n+1}\right)+x^{\zeta\left(\gamma_{2}\right)}$. $\mathcal{A}_{2}\left(\Psi^{n+1}\right)-\mathcal{B}\left(P^{n}, \Psi^{n}\right)$ shows that $X>0$ exists and is unique as soon as $\gamma_{k}>1, P^{n}>0, \Psi_{i}^{n}>0$ and $\Psi_{i}^{n+1}>0$.

\subsubsection{Conservative scheme in volume}

By using the relation (65)(b), we obtain an operator $\wp$ defined with (78) and with

$$
\left\{\begin{array}{l}
\mathcal{A}_{1}(\Psi)=R_{1} \cdot \sum_{i=I^{a}+1}^{I^{b}} \Psi_{i} \\
\mathcal{A}_{2}(\Psi)=R_{2} \cdot\left(\sum_{i=1}^{I^{a}} \Psi_{i}+\sum_{I^{b}+1}^{i_{\max }} \Psi_{i}\right) \\
\mathcal{B}(P, \Psi)=P^{\zeta\left(\gamma_{1}\right)} \cdot \mathcal{A}_{1}(\Psi)+P^{\zeta\left(\gamma_{2}\right)} \cdot \mathcal{A}_{2}(\Psi), \\
\zeta(\gamma)=-\frac{1}{\gamma} .
\end{array}\right.
$$

This operator $\wp$ is similar to the one defined with (78)-(79) but, now, we obtain a scheme which is conservative in volume and non-conservative in energy. We also verify that the function $x \mapsto x^{\zeta\left(\gamma_{1}\right)} \cdot \mathcal{A}_{1}\left(\Psi^{n+1}\right)+x^{\zeta\left(\gamma_{2}\right)}$. $\mathcal{A}_{2}\left(\Psi^{n+1}\right)-\mathcal{B}\left(P^{n}, \Psi^{n}\right)$ admits an unique $X>0$ as soon as $\gamma_{k}>1, P^{n}>0, \Psi_{i}^{n}>0$ and $\Psi_{i}^{n+1}>0$.

\subsubsection{Non-conservative scheme}

The difficulty in the two previous schemes is that we have to solve a non-linear equation at each time step. A more simple scheme is the one defines with

$$
P^{n+1} \equiv \wp\left(P^{n}, \Psi^{n}, \Psi^{n+1}\right)=\wp\left(P^{n}, \Psi^{n}\right)=P^{n}+\Delta t \cdot \mathcal{P}^{\prime}\left(P^{n}, \Psi^{n}\right)
$$

where $\mathcal{P}^{\prime}\left(P^{n}, \Psi^{n}\right)$ is given by $(77)$. The price to pay is that the scheme cannot be conservative in energy and in volume, and that we have to introduce a stability criteria to be sure that $P^{n+1}>0$. Nevertheless, numerical 
results show that this stability criteria is verified as soon as the stability criteria coming from the explicit scheme $(70)(b)$ is verified.

\subsection{Entropic charactere of the scheme}

The numerical scheme verifies the following theorem:

Theorem 5.1. Let us suppose that the initial condition are such that $0<P^{0}<+\infty, \min _{i} \Psi_{i}^{0}>0$ and $\max _{i} \Psi_{i}^{0}<+\infty$. Then, under the stability criteria

$$
\Delta t<\frac{\min _{i} C_{p, i}}{2 \max _{i} \eta_{i}^{n}} \Delta m^{2},
$$

the numerical scheme defined with (70)-(77) verifies

$$
\sum_{i} C_{p, i} \log \Psi_{i}^{n+1} \Delta m \geq \sum_{i} C_{p, i} \log \Psi_{i}^{n} \Delta m
$$

which implies that

$$
\inf _{n, i} \Psi_{i}^{n}>0
$$

Moreover, the inequality (83) is an equality if and only if the system has reached an equilibrium at time $t^{n}$.

At last, when the thermodynamic pressure $P^{n+1}$ is computed with the scheme (78)-(79), we have

$$
\left\{\begin{array}{l}
P^{n} \geq 0, \quad \sup _{n} P^{n}<+\infty, \\
T_{i}^{n} \geq 0, \quad \sup _{n, i} T_{i}^{n}<+\infty .
\end{array}\right.
$$

And when the thermodynamic pressure $P^{n+1}$ is computed with the scheme (78)-(80), we obtain

$$
\inf _{n} P^{n}>0 \quad \text { and } \quad \inf _{n, i} \rho_{i}^{n}>0
$$

The proof of Theorem 5.1 is written in Appendix. Let us note that we have the $l^{\infty}$-stability for the variable $\left(P,\left\{T_{i}\right\}\right)$ only when the scheme is conservative in energy.

\subsection{Convergence of the scheme in the semi-discrete case}

To obtain the convergence of the scheme (70)-(77) toward an equilibrium, we have to prove that the entropy $\mathcal{S}^{n} \equiv-\sum_{i} C_{p, i} \log \Psi_{i}^{n} \Delta m$ is bounded from below which means that we have to prove that $\sup _{n, i} \Psi_{i}^{n}<+\infty$. This last inequality is equivalent to $\inf _{n} P^{n}>0$ when the scheme is conservative in energy (scheme (78)-(79)) and to $\sup _{n} P^{n}<+\infty$ when the scheme is conservative in volume (scheme (78)-(80)). But, it seems very difficult to

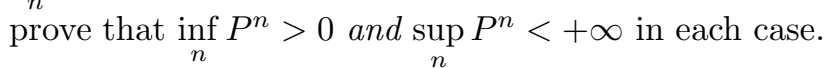

Nevertheless, when the scheme (70)-(77) is continuous in time and discrete in space, it is possible to prove that it is entropic, conservative in energy and conservative in volume. In that case, we obtain that $\inf _{t \geq 0} P(t)>0$ and $\sup _{t \geq 0} P(t)<+\infty$ which allows us to construct a subsequence $\left\{t^{n}\right\}$ such that the semi-discrete scheme converges

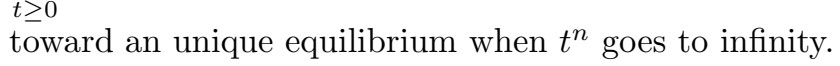




\section{Numerical RESUlts}

We propose numerical results obtained with the numerical scheme described in Section 5 . To simplify the algorithm, the pressure $P^{n+1}$ is computed with the explicit scheme (81). The two perfect gases are defined with the physical constants

$$
\left\{\begin{array} { r l } 
{ \gamma _ { 1 } } & { = 1 , 2 , } \\
{ R _ { 1 } } & { = 0 , 2 , } \\
{ \eta _ { 1 } } & { = 1 }
\end{array} \quad \text { and } \quad \left\{\begin{array}{rl}
\gamma_{2} & =3, \\
R_{2} & =0,7 \\
\eta_{2} & =2
\end{array}\right.\right.
$$

(cf. (48) and (49)). The conductivity $\eta$ is supposed to be a constant $\eta_{k}$ in each domain $\Omega_{k}$ (we recall that $\eta \equiv \rho \lambda$ is the conductivity in the Lagrangian space). The domain $\Omega$ is defined in the Lagrangian space with (51)-(52) by taking $L=1, \Sigma^{a}=-1 / 2$ and $\Sigma^{b}=1 / 2$. The initial thermodynamic pressure is given by $P(t=0)=200$ and the number of meshes $i_{\max }$ is equal to 100 which means that $\Delta m=1 / 50$. At last, the stability criteria ( 82 ) is taken into account in the algorithm through the relaxed criteria $\Delta t=0.4 \times \frac{\min \left(\frac{R_{1} \gamma_{1}}{\gamma_{1}-1}, \frac{R_{2} \gamma_{2}}{\gamma_{2}-1}\right)}{\max \left(\eta_{1}, \eta_{2}\right)} \Delta m^{2}$ which means in the present case that $\Delta t=0.21 \times \Delta m^{2}=8.4 \times 10^{-5}$.

\subsection{Test case $1 \rightarrow$ dilation of the bubble $\Omega_{1}$}

The initial conditions are given by

$$
\begin{cases}m \in \Omega_{2}^{a}: & T(t=0, m)=1,5 \\ m \in \Omega_{1}: & T(t=0, m)=1 \\ m \in \Omega_{2}^{b}: & T(t=0, m)=2\end{cases}
$$

We regularise the initial temperature $T(t=0, m)$ near the interface $\Sigma$ with a regularized Heaviside function in such a way the boundary conditions $(58)(\mathrm{a})(\mathrm{b})\left(\mathrm{a}^{\prime}\right)\left(\mathrm{b}^{\prime}\right)$ are satisfied ${ }^{2}$. We verify that, for these initial conditions, the initial Mach number is less than 0.05. With the formulae (67), we compute the theoretical equilibrium of the continuous DLMN system. We find

$$
T^{\infty} \simeq 1.21, \quad P^{\infty} \simeq 153.90, \quad \rho^{\infty, 1} \simeq 635.95 \quad \text { and } \quad \rho^{\infty, 2} \simeq 181.70
$$

\subsection{Test case $2 \rightarrow$ compression of the bubble $\Omega_{1}$}

The initial conditions are now given by

$$
\begin{cases}m \in \Omega_{2}^{a}: & T(t=0, m)=1.5 \\ m \in \Omega_{1}: & T(t=0, m)=2.5 \\ m \in \Omega_{2}^{b}: & T(t=0, m)=2\end{cases}
$$

After regularization of the initial temperature $T(t=0, m)$, we verify that the initial Mach number is less than 0.05 and we find

$$
T^{\infty} \simeq 2.28, \quad P^{\infty} \simeq 237.84, \quad \rho^{\infty, 1} \simeq 521.58 \quad \text { and } \quad \rho^{\infty, 2} \simeq 149.02
$$

by using again the formulae (67).

\subsection{Numerical results for the test cases 1 and 2}

To illustrate the compression or the dilation of the bubble $\Omega_{1}$, we project the numerical results on the physical space $(t, x)$ by computing the deformation of the mesh with $x_{i+1 / 2}^{n+1}=x_{i+1 / 2}^{n}+\Delta t u_{i+1 / 2}^{n}$ by noting that the velocity $u_{i+1 / 2}^{n}$ is known through the scheme $(70)(\mathrm{d})$. Let us remark that $\left.x_{1 / 2}^{n} \equiv x\right|_{m=-L}=\mathrm{C}^{\text {ste }}$ and that

\footnotetext{
2 Otherwise, the initial velocity $u(t=0, m)$ would be singular on $\Sigma$.
} 
$\left.x_{i_{\max }+1 / 2}^{n} \equiv x\right|_{m=L}=\mathrm{C}^{\text {ste }}$ since $\left.u\right|_{m= \pm L}=0$ (we choose $x_{1 / 2}^{n}=0, x_{i_{\max }+1 / 2}^{n}-x_{1 / 2}^{n}$ being imposed by the initial conditions). Moreover, we normalize $\left[0, x_{i_{\max }+1 / 2}^{n}\right]$ to $[0,1]$ for the visualization of the results.

The first and the second test cases show respectively a dilation and a compression of the bubble $\Omega_{1}$ (Figs. 1-2) and an advection of the bubble $\Omega_{1}$ toward the middle of the domain $\Omega^{3}$. Figures 3-8 show that there is convergence to a stable equilibrium which is very close to the theoretical equilibrium $\left(T^{\infty}, P^{\infty}, u^{\infty}=0\right)(88)$ (89) deduced from the formulae (67) (the equilibrium corresponds to the dot lines in Figs. 3, 4, 7 and 8).

\subsection{What may happen when the thermodynamic Hypothesis 2.1 is not verified}

We now show that the DLMN system may become ill-posed when the equations of state of one of the fluids do not satisfy the thermodynamic Hypothesis 2.1 despite the conductivity, the calorific capacity, the temperature and the pressure are strictly positive. To illustrate this phenomena, we suppose that the equations of state of fluids 1 and 2 are still defined with (48), (49) and (87) except that $\gamma_{2}$ is now equal to -3 . With this choice, the calorific capacity $C_{p, 2}$ is strictly positive (because $\gamma_{2}<-1$ ) and the density is also strictly positive (because the temperature and the pressure remains strictly positive).

We now compare the results when the initial temperature $T(t=0, m)$ in the domain $\Omega_{1}$ is equal to 2.49 and to 2,50 (the other initial conditions are those of Sect. 6.2). If the DLMN system remains well-posed, the differences between these two cases should be tiny. Figure 9 shows that the DLMN system is definitely "chaotic" when the initial temperature in $\Omega_{1}$ is equal to 2.49 . When this initial temperature is equal to 2.5 , it seems that the DLMN system is again asymptotically stable. It is important to note that the entropy $\mathcal{S}(T, P)(t)$ decreases in Figure 10: this is not in contradiction with Figure 9 and with Theorem 5.1. Indeed, despite $\gamma_{2}<0$, the Hypothesis (7)(b) is still verified, the calorific capacity is still strictly positive and the conductivity remains strictly positive. Thus, the inequality (83) is still correct under the stability criteria (82). Nevertheless, since the Hypothesis (7)(a) is not satisfied when $\gamma_{2}<0$, the minimization problem (46)-(47) may not have any solution and the theoretical equilibrium (67) may not be a stable equilibrium of the DLMN system, which seems to be the case when $T(t=0, m)=2,49$ in $\Omega_{1}$.

This example underlines what may happen with the present DLMN system when the equations of state do not satisfy the thermodynamic Hypothesis 2.1 in some region of the $(T, P)$ space. Of course, from a physical point of view, $\gamma_{2}<0$ is not a reasonable value for a perfect gas since it implies that the sound velocity $c_{2}$ is always an imaginary number. Nevertheless, such situations are characteristic of what may happen when phase change phenomena are taking into account.

\section{Conclusion}

We have proposed a Diphasic Low Mach Number (DLMN) system for the modelling of diphasic flows at low mach number. This system, inspired from an earlier work of Majda [21,22], filters out the acoustic waves but keeps all the informations coming from the thermodynamic as the equations of state and the entropy contrary to a standard incompressible diphasic Navier-Stokes system. Thus, the DLMN system is "between" the incompressible diphasic Navier-Stokes system and the compressible diphasic Navier-Stokes system.

This DLMN system has good properties. For example, it predicts the dilation and the compression of a bubble under minimal thermodynamic hypothesis which are verified by a large class of generalized van der Waals equations of state. Moreover, the DLMN system is equivalent to a non-linear heat equation when the two fluids are perfect gases and when the geometry is monodimensional. Using this property, it is possible to build a monodimensional entropic numerical scheme when the two fluids are perfect gases. Moreover, with appropriate modelling hypothesis - again satisfied by a large class of generalized van der Waals equations of state -, the DLMN system degenerates (formally) toward the incompressible Navier-Stokes system for one of the two fluids.

\footnotetext{
${ }^{3}$ The bubble $\Omega_{1}$ has to be on the middle of $\Omega$ when $t \rightarrow+\infty$ since the initial conditions are such that the mass in $\Omega_{2}^{a}$ is equal to the mass in $\Omega_{2}^{b}$.
} 


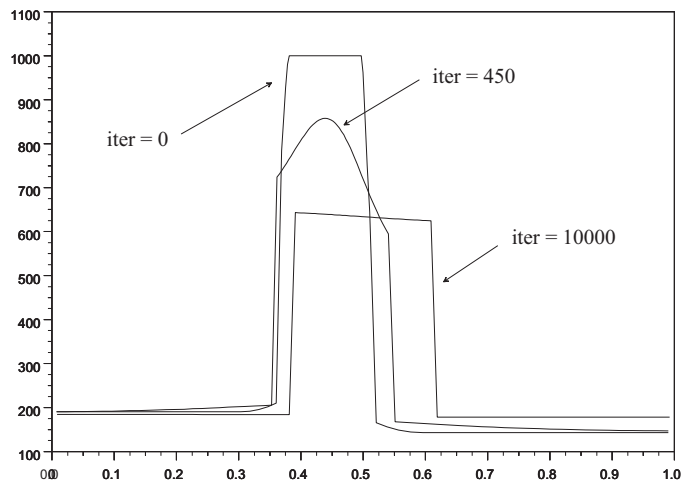

Figure 1. density $\rho(x)$. Test case 1

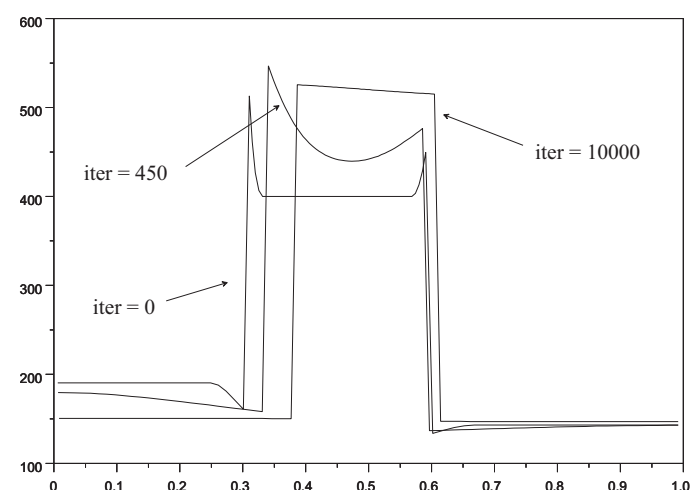

Figure 2. density $\rho(x)$.

Test case 2

Iterations $=0,450$ and 10000.

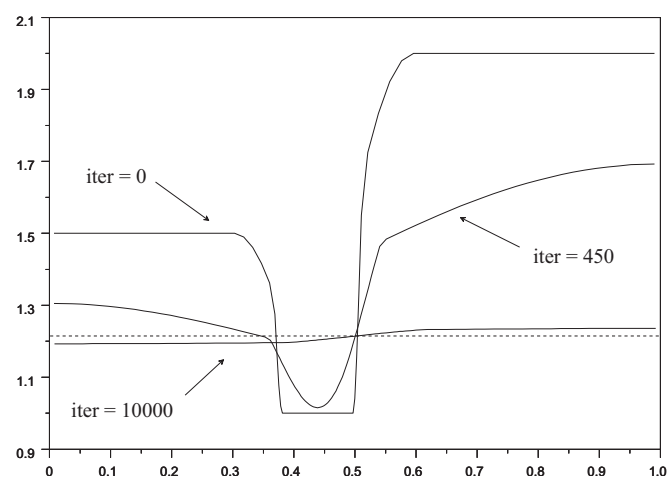

Figure 3 . temperature $T(x)$. Test case 1

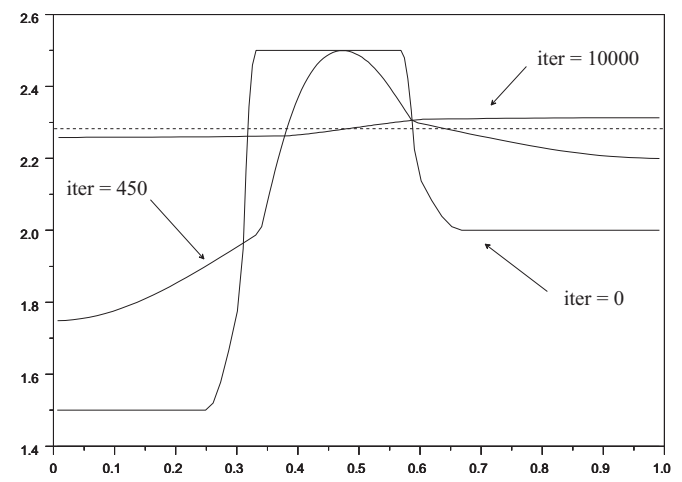

Figure 4 . Temperature $T(x)$. Test case 2

Iterations $=0,450$ and 10000 .

Nevertheless, the present DLMN system does not take into account any gas-liquid phase change phenomena even though it is an important topic in the field of diphasic flows. Moreover, monodimensional simulations are not physically relevant since any coalescence or fragmentation of bubbles is not possible when the space dimension is equal to one. Thus, future works on the DLMN system will have to take into account the thermodynamics of gas-liquid phase change knowing that the liquid phase is better modelled with an ad hoc generalized van der Waals equation of state than with a simple perfect gas equation of state. And bi or tridimensional algorithms will have to be proposed. The level set technic $[16,23,24,26]$ could be applied to the capture of the gas-liquid interface in these algorithms. Preliminary results are proposed in the last chapter of [7] in monodimensional geometry and for perfect gases.

Acknowledgements. This research has been done with the financial support of the DGA (grant CEA-DGA 026000062 ) and with the financial support of the Centre de Recherches Mathématiques of Montréal. I wish also to thank Jacques Segré and Daniel Caruge of CEA, Alain Vincent and Anne Bourlioux of Montréal University for their technical and financial support. 


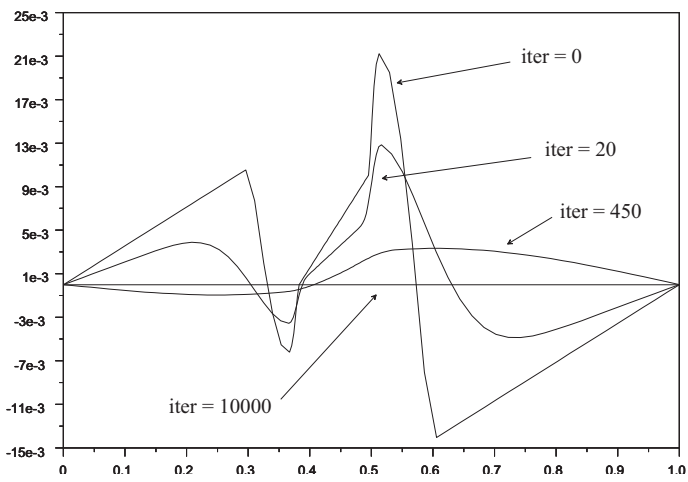

Figure 5. Velocity $u(x)$. Test case 1

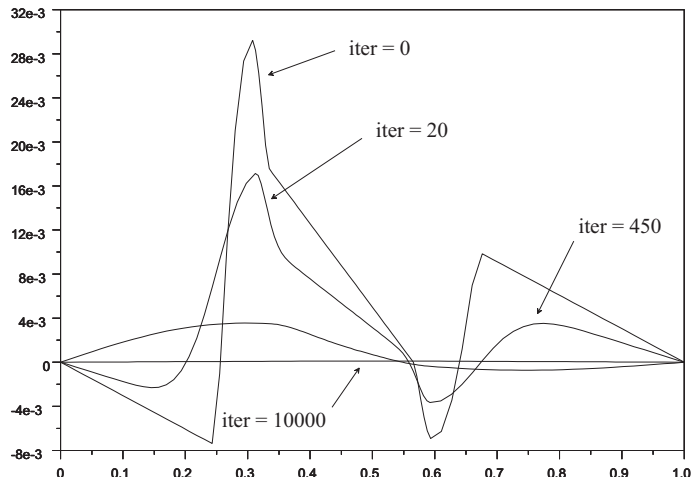

Figure 6. Velocity $u(x)$. Test case 2

Iterations $=0,20,450$ and 10000 .

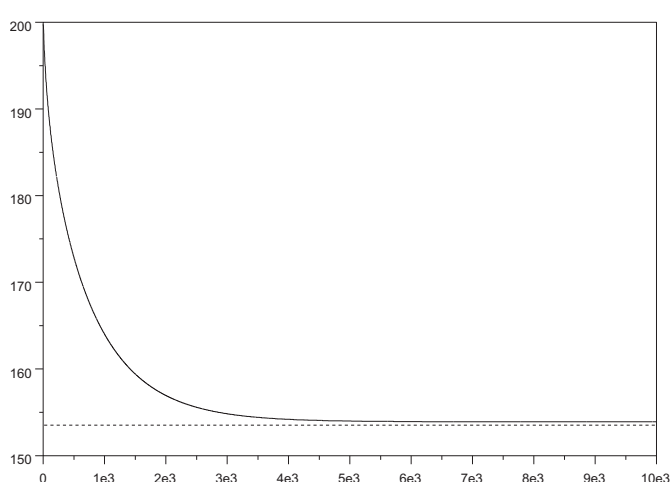

Figure 7. Pressure. Test case 1

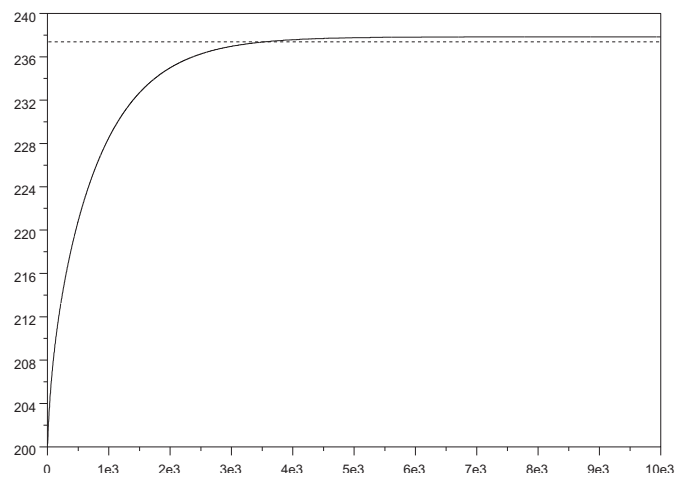

Figure 8. Pressure $P(t)$.

Iterations $=1 \ldots 10000$.

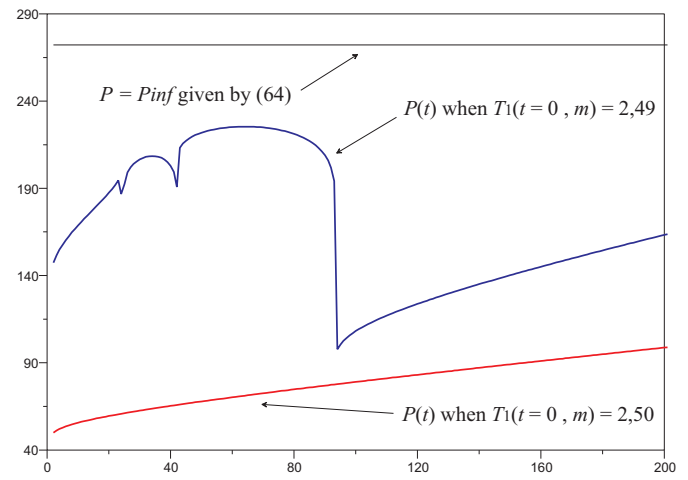

Figure 9. Pressure.

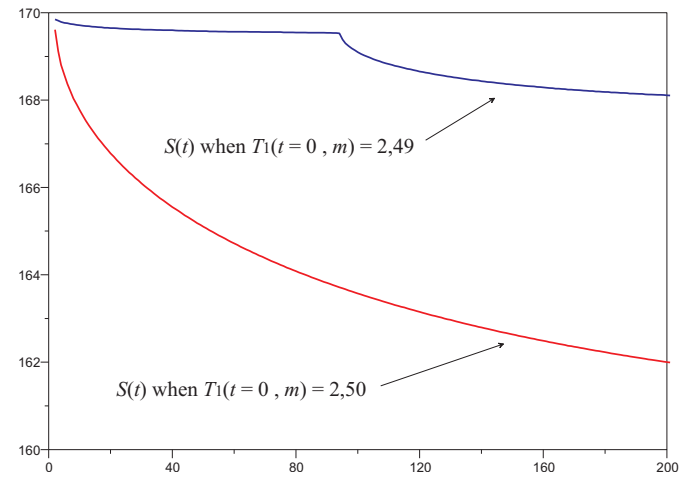

Figure 10. Entropy $\mathcal{S}(T, P)(t)$.

"Chaotic" phenomena when the entropy is not a convex function. 


\section{Appendix. Proof of theorem 5.1}

To simplify the proof, we suppose that the interface $\Sigma$ is equal to the interface $\Sigma^{a}$ which means that $i \in$ $\left\{1, \ldots, I^{a}\right\}$ is related to fluid 2 , and $i \in\left\{I^{a}+1, \ldots, i_{\max }\right\}$ is related to fluid 1 .

\section{A.1. Proof of the inequality (83)}

The proof is classical: it uses the strict convexity of the function $x \mapsto-\log x$. The difference with the classical heat equation lies on the boundary condition (75) on the interface $\Sigma$. For any $i \notin\left\{I^{a}, I^{a}+1\right\}$, it is possible to write the scheme $(70)(\mathrm{b})$ with

$$
\Psi_{i}^{n+1}=\mathcal{A}_{i}^{n} \Psi_{i-1}^{n}+\mathcal{B}_{i}^{n} \Psi_{i}^{n}+\mathcal{C}_{i}^{n} \Psi_{i+1}^{n}
$$

where

$$
\mathcal{A}_{i}^{n}=\frac{\eta_{i-1 / 2}^{n}}{C_{p, i} \Delta m^{2}} \Delta t, \quad \mathcal{B}_{i}^{n}=1-\frac{\eta_{i-1 / 2}^{n}+\eta_{i+1 / 2}^{n}}{C_{p, i} \Delta m^{2}} \Delta t \quad \text { and } \quad \mathcal{C}_{i}^{n}=\frac{\eta_{i+1 / 2}^{n}}{C_{p, i} \Delta m^{2}} \Delta t
$$

(when $i \in\left\{1, i_{\max }\right\}$, we write $\eta_{1 / 2}^{n}=\eta_{i_{\max }+1 / 2}^{n} \equiv 0$ ). And when $i \in\left\{I^{a}, I^{a}+1\right\}$, we deduce from (75) that

$$
\left\{\begin{array}{l}
\Psi_{I^{a}}^{n+1}=\mathcal{A}_{I^{a}}^{n} \Psi_{I^{a}-1}^{n}+\mathcal{B}_{I^{a}}^{n} \Psi_{I^{a}}^{n}+\mathcal{C}_{I^{a}}^{n} P^{n \beta_{2}^{1}} \Psi_{I^{a}+1}^{n} \\
\Psi_{I^{a}+1}^{n+1}=\mathcal{A}_{I^{a}+1}^{n} P^{n-\beta_{2}^{1}} \Psi_{I^{a}}^{n}+\mathcal{B}_{I^{a}+1}^{n} \Psi_{I^{a}+1}^{n}+\mathcal{C}_{I^{a}+1}^{n} \Psi_{I^{a}+2}^{n}
\end{array}\right.
$$

where $\mathcal{A}_{I^{a}}^{n}, \mathcal{B}_{I^{a}}^{n}, \mathcal{C}_{I^{a}}^{n}, \mathcal{A}_{I^{a}+1}^{n}, \mathcal{B}_{I^{a}+1}^{n}$ and $\mathcal{C}_{I^{a}+1}^{n}$ are given by (91). Moreover, it is not difficult to find that

$$
\begin{aligned}
\sum_{i} C_{p, i} \log \Psi_{i}^{n} \Delta m= & C_{p, 1}\left(\mathcal{B}_{1}^{n} \log \Psi_{1}^{n}+\mathcal{C}_{1}^{n} \log \Psi_{2}^{n}\right) \Delta m+C_{p, i_{\max }}\left(\mathcal{A}_{i_{\max }}^{n} \log \Psi_{i_{\max }-1}^{n}+\mathcal{B}_{i_{\max }}^{n} \log \Psi_{i_{\max }}^{n}\right) \Delta m \\
& +\sum_{i \notin\left\{1, I^{a}, I^{a}+1, i_{\max }\right\}} C_{p, i}\left(\mathcal{A}_{i}^{n} \log \Psi_{i-1}^{n}+\mathcal{B}_{i}^{n} \log \Psi_{i}^{n}+\mathcal{C}_{i}^{n} \log \Psi_{i+1}^{n}\right) \Delta m \\
& +C_{p, I^{a}}\left[\mathcal{A}_{I^{a}}^{n} \log \Psi_{I^{a}-1}^{n}+\mathcal{B}_{I^{a}}^{n} \log \Psi_{I^{a}}^{n}+\mathcal{C}_{I^{a}}^{n} \log \left(P^{n \beta_{2}^{1}} \Psi_{I^{a}+1}^{n}\right)\right] \Delta m \\
& +C_{p, I^{a}+1}\left[\mathcal{A}_{I^{a}+1}^{n} \log \left(P^{n-\beta_{2}^{1}} \Psi_{I^{a}}^{n}\right)+\mathcal{B}_{I^{a}+1}^{n} \log \Psi_{I^{a}+1}^{n}+\mathcal{C}_{I^{a}+1}^{n} \log \Psi_{I^{a}+2}^{n}\right] \Delta m
\end{aligned}
$$

by using the fact that $\forall i: \mathcal{A}_{i+1}^{n}+\mathcal{B}_{i}^{n}+\mathcal{C}_{i-1}^{n}=1$ and that $C_{p, I^{a}} \mathcal{C}_{I^{a}}^{n} \log \left(P^{n \beta_{2}^{1}}\right)+C_{p, I^{a}+1} \mathcal{A}_{I^{a}+1}^{n} \log \left(P^{n-\beta_{2}^{1}}\right)=0$. Moreover, $\forall i: \mathcal{A}_{i}^{n}+\mathcal{B}_{i}^{n}+\mathcal{C}_{i}^{n}=1, \mathcal{A}_{i}^{n} \geq 0, \mathcal{C}_{i}^{n} \geq 0$, and, under the stability criteria

$$
\Delta t<\frac{\min _{i} C_{p, i}}{2 \max _{i} \eta_{i}^{n}} \Delta m^{2}
$$

we have also $\mathcal{B}_{i}^{n} \geq 0$. Thus, the relations (90) shows that $\Psi_{i}^{n+1}$ is a convex combinaison of $\Psi_{i-1}^{n}, \Psi_{i}^{n}$ and $\Psi_{i+1}^{n}$ for any $i \notin\left\{I^{a}, I^{a}+1\right\}$. In the same way, (92) shows that, under the stability criteria $(94), \Psi_{I^{a}}^{n+1}$ is a convex combinaison of $\Psi_{I^{a}-1}^{n}, \Psi_{I^{a}}^{n}$ and $P^{\beta_{2}^{1}} \Psi_{I^{a}+1}^{n}$, and shows also that $\Psi_{I^{a}+1}^{n+1}$ is a convex combinaison of $P^{-\beta_{2}^{1}} \Psi_{I^{a}}^{n}, \Psi_{I^{a}+1}^{n}$ and $\Psi_{I^{a}+2}^{n}$. Since $x \mapsto-\log x$ is a strictly convex function, we deduce from (93) that $\sum_{i} C_{p, i} \log \Psi_{i}^{n+1} \Delta m \geq \sum_{i} C_{p, i} \log \Psi_{i}^{n} \Delta m$ under the stability criteria (94) and that $\sum_{i} C_{p, i} \log \Psi_{i}^{n+1} \Delta m=$ $\sum_{i} C_{p, i} \log \Psi_{i}^{n} \Delta m$ if and only if it exists $\Psi^{\infty}>0$ such that

$$
\forall i \in\left\{1, \ldots, I^{a}\right\}: \quad \Psi_{i}^{n}=\Psi^{\infty} \quad \text { and } \quad \forall i \in\left\{I^{a}+1, \ldots, i_{\max }\right\}: \quad \Psi_{i}^{n}=P^{-\beta_{2}^{1}} \Psi^{\infty}
$$

which is equivalent to $\forall i: \Psi_{i}^{n+1}=\Psi_{i}^{n}$. 


\section{A.2. Proof of inequalities (85) and (86)}

Let us suppose that $P^{n+1}$ is computed with (78)-(79). Since the initial energy $\mathcal{B}\left(P^{0}, \Psi^{0}\right)$ is bounded when $P^{0}<+\infty$ and $\max _{i} \Psi_{i}^{0}<+\infty$, we obtain that $\sup _{i, n}\left(\Psi_{i}^{n} \cdot P^{n \frac{\gamma_{i}-1}{\gamma_{i}}}\right)<+\infty$. And since $\inf _{n, i} \Psi_{i}^{n}>0$ because of the entropic charactere of the scheme, we finally find that $\sup _{n} P^{n}<+\infty$. At last, we have $\sup _{n, i} T_{i}^{n}<+\infty$ since $T_{i}^{n}=\Psi_{i}^{n} \cdot P^{n \frac{\gamma_{i}-1}{\gamma_{i}}}$. The proof is similar when $P^{n+1}$ is computed with the scheme (78)-(80). Indeed, since the volume is bounded, we obtain that $\sup _{i, n}\left(\Psi_{i}^{n} \cdot P^{n-\frac{1}{\gamma_{i}}}\right)<+\infty$ which implies that $\inf _{n} P^{n}>0$ since $\inf _{n, i} \Psi_{i}^{n}>0$. We conclude by noting that $\rho_{i}^{n}=\left(R_{i} \Psi_{i}^{n} \cdot P^{n-\frac{1}{\gamma_{i}}}\right)^{-1}$.

\section{REFERENCES}

[1] R. Abgrall, R. Saurel, A multiphase Godunov method for compressible multifluid and multiphase flows. J. Comput. Phys. 150 425-467 (1999).

[2] G. Allaire, S. Clerc and S. Kokh, A five-equation model for the numerical simulation of interfaces in two-phase flows. C. R. Acad. Sci. Paris Ser. I 331 (2000) 1017-1022.

[3] G. Allaire, S. Clerc and S. Kokh, A five-equation model for the simulation of interfaces between compressible fluids. J. Comput. Phys. 181 (2002) 577-616.

[4] Y.-H. Choi, C.L. Merkle, The Application of Preconditioning in Viscous Flows. J. Comput. Phys. 105 (1993) $207-223$.

[5] A.J. Chorin and J.E. Mardsen, A Mathematical Introduction to Fluid Mechanics. Springer-Verlag (1979).

[6] S. Dellacherie, On relaxation schemes for the multicomponent Euler system. ESAIM: M2AN 37 (2003) 909-936.

[7] S. Dellacherie, Dérivation du système diphasique bas Mach. Simulation numérique en géométrie monodimensionnelle. CEA report, ref. CEA-R-6046 (2004).

[8] S. Dellacherie and A. Vincent, Zero Mach Number Diphasic Equations for the Simulation of Water-Vapor High Pressure Flows, in Proc. of the 11th conference of the CFD Society of Canada, Vancouver (2003) 248-255.

[9] P. Embid, Well-posedness of the nonlinear equations for zero Mach number combustion. Comm. Partial Differential Equations 12 (1987) 1227-1283.

[10] D. Gueyffier, J. Li, A. Nadim, R. Scardovelli and S. Zaleski, Volume-of-Fluid interface tracking with smoothed surface stress methods for three-dimensional flows. J. Comput. Phys. 152 (1999) 423-456.

[11] H. Guillard and A. Murrone, On the behavior of upwind schemes in the low Mach number limit: II. Godunov type schemes. Comput. Fluids 33 (2004) 655-675.

[12] H. Guillard and C. Viozat, On the behaviour of upwind schemes in the low Mach number limit. Comput. Fluids 28 (1999) $63-86$.

[13] F.H. Harlow and J.E. Welch, Numerical calculation of time-dependent viscous incompressible flow of fluid with free interface. Phys. Fluids 8 (1965) 2182-2189.

[14] D. Jamet, O. Lebaigue, N. Coutris and J.M. Delhaye, The second gradient method for the direct numerical simulation of liquid-vapor flows with phase change. J. Comput. Phys. 169 (2001) 624-651.

[15] D. Juric and G. Tryggvason, Computations of boiling flows. Int. J. Multiphase Flow 24 (1998) 387-410.

[16] S. Kokh, Aspects numériques et théoriques de la modélisation des écoulements diphasiques compressibles par des méthodes de capture d'interface. Ph.D. thesis of Paris VI University (2001).

[17] B. Lafaurie, C. Nardone, R. Scardovelli, S. Zaleski and G. Zanetti, Modelling merging and fragmentation in multiphase flows with SURFER. J. Comput. Phys. 113 (1994) 134-147.

[18] F. Lagoutière, Modélisation mathématique et résolution numérique de problèmes de fluides compressibles à plusieurs constituants. Ph.D. thesis of Paris VI University (2000).

[19] D. Lakehal, M. Meier and M. Fulgosi, Interface tracking towards the direct numerical simulation of heat and mass transfer in multiphase flow. Internat. J. Heat Fluid Flow 23 (2002) 242-257.

[20] J.M. Le Corre, E. Hervieu, M. Ishii and J.M. Delhaye, Benchmarking and improvements of measurement techniques for local time-averaged two-phase flow parameters. Fourth International Conference on Multiphase Flows (ICMF 2001), New-Orleans, USA (2001).

[21] A. Majda, Equations for low mach number combustion. Center of Pure and Applied Mathematics, University of California at Berkeley, report No. 112 (1982).

[22] A. Majda and J.A. Sethian, The derivation and numerical solution of the equations for zero Mach number combustion. Combust. Sci. Tech. 42 (1985) 185-205.

[23] W. Mulder, S. Osher and J.A. Sethian, Computing interface motion in compressible gas dynamics. J. Comput. Phys. 100 (1992) 209-228. 
[24] S. Osher, M. Sussman and P. Smereka, A level set approach for computing solutions to incompressible two-phase flow. J. Comput. Phys. 114 (1994) 146-159.

[25] S. Paolucci, On the filtering of sound from the Navier-Stokes equations. Sandia National Laboratories report SAND82-8257 (1982).

[26] J.A. Sethian, Level Set Methods. Cambridge Monographs on Applied and Computational Mathematics, Cambridge University Press (1996).

[27] K.M. Shyue, A fluid-mixture type algorithm for compressible multicomponent flow with van der Waals equation of state. J. Comput. Phys. 156 (1999) 43-88.

[28] G. Tryggvasson and S.O. Unverdi, A front-tracking method for viscous, incompressible, multi-fluid flows. J. Comput. Phys. $100(1992) 25-37$.

[29] E. Turkel, Review of preconditioning methods for fluid dynamics. Appl. Numer. Math. 12 (1993) 257-284.

[30] S.W.J. Welch and J. Wilson, A volume of fluid based method for fluid flows with phase change. J. Comput. Phys. 160 (2000) $662-682$.

To access this journal online:

www.edpsciences.org 